\title{
Empowered Hybrid Parent Selection for Improving Network Lifetime, PDR, and Latency in Smart Grid
}

\author{
Kanabadee Srisomboon $(\mathbb{D}$, Tinnaphob Dindam $(\mathbb{D}$, and Wilaiporn Lee \\ Communication and Computer Network Research Group (C2NRG), Electrical Engineering, \\ Department of Electrical and Computer Engineering, Faculty of Engineering, \\ King Mongkut's University of Technology North Bangkok, Bangkok, Thailand \\ Correspondence should be addressed to Wilaiporn Lee; wilaiporn.l@eng.kmutnb.ac.th
}

Received 20 January 2021; Accepted 13 May 2021; Published 25 May 2021

Academic Editor: Mohammad Mahdi Abaei

Copyright $\odot 2021$ Kanabadee Srisomboon et al. This is an open access article distributed under the Creative Commons Attribution License, which permits unrestricted use, distribution, and reproduction in any medium, provided the original work is properly cited.

\begin{abstract}
To support the constraints of smart meters-low power and memory-of AMI network, RPL is considered as the most suitable routing protocol to be implemented in practice. Network lifetime, PDR, and latency are the critical issues to be focused on and addressed. Generally, single parent selection scheme cannot satisfy all expected performance requirements of RPL based on AMI network due to tradeoff between workload balancing and transmission performance, PDR and latency. Moreover, the single parent also suffers from the package size and transmission range. Then, multiparent solution is proposed to overcome these demerits using multipath transmission strategy. Although the existing multiparent solutions, MELT and MAHP, overcome the issue of transmission performance, they present low network lifetime since multiparent solution consumes high energy in data transmission. In this paper, we propose an "empowered hybrid parent selection (EHPS)" that exploits the merits of multiparent solution and the single parent with cognitive radio technology in a hybridizing scheme. To split the data packet efficiently under multipath transmission strategy, a fuzzy AHP (FAHP) is adopted; therefore, EHPS balances the workload effectively and maximizes the network lifetime over long transmission range and large data size. Moreover, by exploiting cognitive radio, EHPS is flexible to the transmission range and data size since it achieves the highest transmission performance, highest PDR, and lowest latency among others, while maintaining high network lifetime.
\end{abstract}

\section{Introduction}

With increasing electrical demands, the electrical grid shows a tendency to be managed more efficiently, which cannot be achieved by the conventional strategy based on a centralized and manual management system. To alleviate the impairment of conventional grid, a smart grid is being recognized as the next generation of electrical grid which corroborates communication and data processing technology with the electrical grid. Therefore, smart grid achieves system efficiency, reliability, and robustness of electrical generation and distribution system. The advanced metering infrastructure (AMI) [1-5] is the crucial application of the smart grid which manages information between utility and clients, e.g., smart meters, through a local access point (data concentrator unit: DCU) using the two-way communication method. Once the information can be exchanged between smart meters and the DCU in both directions and in real time, the smart grid is recognized as the self-monitoring and self-healing system since simple troubleshooting and repairing can be performed automatically without technician intervention.

In an AMI network, a number of low power and memory devices, smart meters, are interconnected in a multihop manner through modern communication technology. The routing protocol is considered as a crucial function in the AMI network that is used to ensure high communication performance in terms of packet delivery ratio (PDR) and latency. The routing protocol for low power and lossy networks (RPL) [6-13] is considered as the most suitable routing protocol for AMI network according to its characteristics as it has the ability to deal with lossy links, support 
communication in a point-to-multipoint and multipoint-topoint manner, and provide a low energy consumption and low bandwidth usage due to low signaling overheads [7]. Once the battery and memory are the limitation of the smart meters [8], not only PDR and latency are determined, but also the meter workload should be taken into account to prolong a network lifetime and prevent information loss due to a fast battery depletion issue.

Based on the RPL routing scheme, a smart meter forwards the data to the DCU through several relay nodes, i.e., the neighbor smart meters advertised by lower rank level. In RPL, the relay node is known as a parent. If the workload is not considered, the smart meter with a high workload will deplete fast and before the maintenance session is performed, which refers to an early depletion node. Generally, the network lifetime is determined by the early depletion node. Therefore, the utility loses the information to be used for the electrical production planning and electricity billing application. Then, each meter lifetime needs to be monitored carefully and individually. On the other hand, to achieve the objective of smart grid, a high transmission performance, i.e., PDR and latency, is required.

PDR, the success data transmission rate, should be high while the latency should be low to achieve a real-time application [14]. These two metrics are affected by the quality of the selected link of parent meters. If the link quality of parent meter is low, the data packets may drop during the transmission; then, the data retransmission is required. Therefore, the smart meter must consume extra energy for the data retransmission.

Parent selection is the vital function of the RPL that plays an important role in avoiding the early depletion node while maintaining the performance of the transmission. Currently, the parent selection can be categorized into single selection [15-23] and multiparent solution scheme [24-26]. Traditionally, parent selection of RPL determines the single immediate parent by considering only a single metric, i.e., residual energy or expected transmission count (ETX). By considering only the ETX [15-18], only the parent with the highest quality is selected; then, it achieves a high PDR and low latency. However, the batteries of the high quality parents deplete much faster than the others. Therefore, the early depletion node occurs. On the other hand, although the parent selection based on residual energy $[19,20]$ balances the workload perfectly, high PDR and low latency cannot be met since the low quality parent is selected. Later, several techniques [21-23] are proposed to address the issues by taking energy into account. However, they cannot satisfy overall performance.

On the contrary, the rate of PDR is affected by two important factors: link quality and data package size. The link quality degrades on the transmission channel characteristic and the transmission range. On the other hand, the data package size depends on the application of AMI; i.e., the package size for energy usage of household is much smaller than that for energy usage of manufacturing, which causes a decrease in the PDR rate and an increase in the latency. As stated in RFC standard [13], once the single parent suffers from the low rate of PDR, the multiple parent needs to be considered to address the issue. Moreover, depending on convergecast traffic of single parent scheme, there is always a tradeoff between the transmission performance and the load balancing ability since all of the data from a single node will be transmitted to the immediate parent. Multiparent solution is proposed to address this tradeoff issue by splitting the data into small packets and forwarding them to a number of parents. The multiparent based on ELT (MELT) [26] is proposed by splitting the parents' workload on an estimated the expected lifetime (ELT) [23] according to the ELT of bottleneck parents. However, the workload of MELT splits on the fixed step; therefore, the workload of each parent is not balanced comprehensively and effectively. Later, an analytic hierarchy process (AHP) [27-31] is utilized for multiparent selection (MAHP) [32] to improve the performance of MELT by considering ETX, node remaining energy, and hop count (HC) as the main criteria. However, under fixed influence weights of criteria, MAHP cannot achieve high network lifetime since the meters with different rank have different workloads. Therefore, the influence weight of energy-efficient criteria should be varied on the ranking.

In this paper, we propose the empowered hybrid parent selection (EHPS) algorithm of RPL for AMI network. The multiparent solution with fuzzy analytic hierarchy process (FAHP) [33-35] and the cognitive radio [36, 37] are exploited under hybridizing scheme to achieve high performance of the three crucial metrics: network lifetime, PDR, and latency. Cognitive radio is the advanced communication technology that is proposed to deal with the underutilization of spectrum resources. The unlicensed user is permitted to utilize the available licensed channel while causing unnoticeable interference to the licensed user. Therefore, the meter should be considered as the cognitive device that can monitor the status of surrounding communication channels and can adapt its parameter for using on the selected channel.

The contribution of this paper is threefold. First, network lifetime is maximized through hybrid parent selection algorithm. The quality of parents is comprehensively analyzed through the FAHP, which is an extended scheme of an analytic hierarchy process (AHP) with the weight adapting scheme where ETX, ELT, and expected transmission time (ETT) are determined as the main criteria. The meter's ranking is considered in adapting the influence of the energy efficiency since the meters advertised by the lower rank come at a cost of high workload; i.e., the lower rank meters have to forward the data from their children. Then, the appropriate size of data package is forwarded to each parent on its quality. Therefore, the workload of each parent is balanced effectively, and the network lifetime is prolonged.

Second, the issue of low PDR under long transmission range is addressed by hybridizing the merits of multiple parents and single parent with cognitive radio technology. Through multiparent solution, the PDR is improved by forwarding a number of small packets to $k$ parents since the transmission error rate is affected by the size of transmitted data. Moreover, under long transmission range, multiple parents cannot achieve high performance of PDR and energy 
efficiency because the transmission error rate also increases on the distance. Therefore, we exploit the cognitive radio technology on the single parent scheme to address the transmission range issue. Under cognitive radio technology, the meter forwards data on available licensed channels opportunistically. Although the meters should consume higher transmission power as compared to the original Zigbee [38] technology of RPL, we achieve high energy efficiency and high PDR since the meter on Zigbee channel wastes the energy on performing retransmission which also gives low PDR. It should be noted that the single parent with cognitive radio cannot achieve high energy-efficient performance under short transmission range since multiple parents can present high PDR at short transmission range. Once the single parent with cognitive radio consumes the energy usefully, the EHPS presents the highest network lifetime under long transmission range.

Third, the EHPS achieves the lowest latency since it presents high PDR under hybridizing scheme. Under low transmission range, a low latency is achieved by using multiparent solution while the EHPS maintains low latency under long transmission range using the cognitive radio.

The remainder of this paper is organized as follows. In Section 2, the details of RPL and parent selection methods are introduced. The issues of parent selection are described in Section 3. In Section 4, EHPS algorithm is proposed and described in detail. The simulation results and the performance evaluation are presented in Section 5. Conclusion is presented in the final section.

\section{Related Works}

2.1. IPv6 Routing Protocol for Low Power and Lossy Networks $(R P L)$. RPL [6-13] is the routing standard for the lossy network comprising low power and memory devices. It is considered as the most suitable routing protocol to be implemented with the smart meters of AMI network since the RPL has the ability to deal with lossy links, support communication in a point-to-multipoint and multipoint-topoint manner, and provide a low energy consumption and low bandwidth usage due to low signaling overheads [9].

The key characteristic of RPL is the interconnection with loop avoiding of RPL network-directed acyclic graphs (DAG). Through distance vector base routing, the RPL constructs a rooted $k$-ary tree routing topology, destinationoriented directed acyclic graphs (DODAG), according to distance vector where the role of each node is advertised by the rank. In $k$-ary tree topology, $k$ is the number of children for each parent. As illustrated in Figure 1, $k$ is set to 3, so each parent has 3 children.

In the DODAG construction and maintenance process, a root node-border router or data concentrator (DCU) of AMI network-broadcasts the DAG information option (DIO) message, which contains DODAG identifier, current rank of the node, objective function (OF), and additional information for path computation to neighbors. The neighbors who receive the broadcasted DIO message use the received information to update their rank level, select the immediate parent, and join the DODAG. Therefore, the neighbor who selects the broadcasted DIO message as the immediate parent is considered as the child node. Then, the child node sends the feedback to its immediate parent using destination advertisement object (DAO) message.

As illustrated in Figure 2, firstly, the root node broadcasts DIO message to node 2 , node 3 , and node 4 . Then, these nodes update their rank level to "rank 1" and they select the route node as the immediate parent by sending the DAO message to the route node. Once node 2 , node 3 , and node 4 complete their DODAG joining process, they will broadcast the DIO message to other neighbors, node 5 to node 9 , for further DODAG construction. On the other hand, if a node needs to join the DODAG or needs to perform local route maintenance when batteries of all candidate parents deplete, the node exploits DODAG information solicitation (DIS) to request route maintenance. Therefore, the nodes who receive DIS message will broadcast DIO message to neighbors and the requested node. It can be noticed that the level of rank of a node is assigned according to the distance vector to the root node. The nodes with highest rank can transmit their information to the root node in upward direction. To avoid information looping, the node in the topology is not allowed to transmit the DAO message to neighbors with the same rank.

RPL also supports data transmission in an upward direction; therefore, the smart meters can forward the information, such as energy usage, to the DCU via ancestor meters. In the upward data transmission process, the child meter exploits DAO message to transmit its information to the DCU in a unicast manner. The DAO message contains the generated information and the target parents. The immediate parent who receives the DAO message will send an acknowledgment message to the child node, generate a new DAO message, and send the new DAO message containing the message generated by the child to its parent. The process is repeated until the generated message reaches the target parent. Therefore, the framework of RPL is suitable to be implemented in the smart meters of AMI network. Once the memory and battery are considered as the limitation of the meters [8], not only the packet delivery ratio (PDR) and latency are determined, but also the energy efficiency must be taken into account.

2.2. Parent Selection. Parent selection plays the important role in achieving a high requirement of data transmission of AMI application where the PDR should be as high as 0.98 [14]. Generally, parent selections can be categorized into single parent and multiparent method. Traditionally, the first standard of RPL selects the parent based on a single metric, expected transmission count (ETX) or residual energy. In this paper, we consider that the parent selection based on ETX is called "RPL." The RPL selects the parent by determining the ETX metric where ETX is given as

$$
\operatorname{ETX}=\frac{1}{\left(D_{f} \times D_{r}\right)}=\frac{1}{1-p},
$$

where $D_{f}$ is the probability that the packets are received by the neighbor, $D_{r}$ is the probability that the acknowledgment 


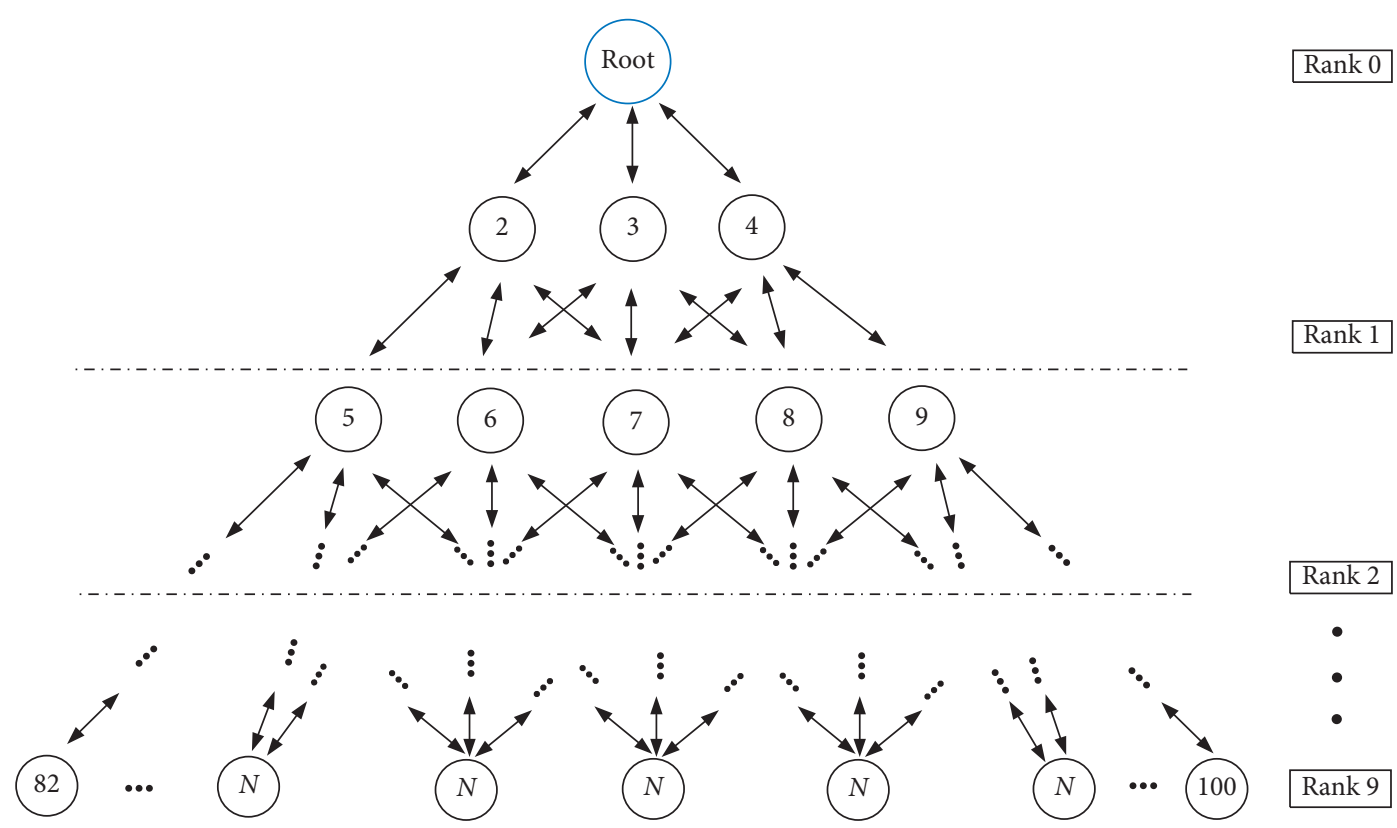

FIGURE 1: 3-ary tree topology of RPL network.

packet is successfully received, and $p$ is the error data transmission rate.

It can be noticed that ETX is used to determine the quality of parents from the perspective of the PDR. The parent will be declared to be of high quality if the ETX is as low as 1 . If the ETX is greater than 1, the quality of the parent becomes worse. Even if the RPL achieves high PDR, it does not take the workload balancing into account since the child selects only the highest quality parent to be the immediate parent. The parent selection of the RPL is illustrated in Figure 3 where the immediate parent is selected based on the lowest ETX.

Later, the alternative metrics are considered for single parent selection scheme. However, they cannot overcome the tradeoff between the transmission performance and the energy consumption. Moreover, they cannot achieve high transmission performance affected by the data package size and transmission range. Therefore, RFC standard [13] stated that multiparent, multipath routing scheme should be considered to address the single parent issues. The multipath routing scheme splits the data into small packets and forwards them to a number of parents. Multiparent solution based on ELT (MELT) [26] is proposed to improve the workload balancing while maintaining the PDR at high rate by considering the expected lifetime of child node according to the ELT of bottleneck parents. The bottleneck meter has a noticeably high workload and seems to be the first node whose battery depletes. The expected lifetime (ELT) of a node $(\mathrm{N})$ is considered as

$$
\operatorname{ELT}(N)=\frac{E_{\text {res }}(N)}{\left(T_{N} \times \operatorname{ETX}(N, P) / \text { DATA_RATE }\right) \times P_{\text {RadioTX }}(N)},
$$

where $T_{N}$ is the traffic generated by a considered meter and its children, $\operatorname{ETX}(N, P)$ is the ETX of the meter and its considered parent (P), and DATA_RATE is the transmission rate.

It should be mentioned that the residual energy $\left(E_{\text {res }}(N)\right)$ can be calculated by

$$
E_{\text {res }}(N)=\text { Battery }-E_{\text {cons }}(N) \text {, }
$$

where the energy consumption $\left(E_{\text {cons }}(N)\right)$ is given as

$$
\begin{aligned}
E_{\text {cons }}(N)= & T_{\mathrm{CPU}} \times P_{\mathrm{CPU}}+T_{\text {RadioRX }}(N) \times P_{\text {RadioRX }}(N) \\
& +T_{\text {RadioTX }}(N) \times P_{\text {RadioTX }}(N),
\end{aligned}
$$

where $T_{\mathrm{CPU}}$ is the CPU processing time, $P_{\mathrm{CPU}}$ is the power consumption of CPU, $T_{\text {RadioRX }}(N)$ is the receiver processing time, $P_{\text {RadioRX }}(N)$ is the power consumption of receiver, $T_{\text {RadioTX }}(N)$ is the transmitter processing time, and $P_{\text {RadiotX }}(N)$ is the power consumption of transmitter.

Therefore, parents' workload is balanced according to the expected lifetime of the node and the bottlenecks through the traffic ratio $\left(\alpha_{p}\right)$. Generally, the bottleneck nodes are the nodes at rank 1 . The load balancing algorithm of MELT is shown in Algorithm 1.

To determine the traffic ratio $\left(\alpha_{p}\right)$, node $N$ determines the best parent iteratively to assign the highest traffic ratio. Firstly, the minimum ELT of node $N$ and bottlenecks are estimated by increasing $\alpha_{p}$ of considered parent individually with the load balancing step $(\gamma)$. Secondly, the best parent with the maximum expected lifetime (ELT) is determined. Thirdly, node $N$ decreases $\alpha_{p}$ of considered parent and increases $\alpha_{p}$ of other parents to investigate the expected lifetime (ELT). Finally, the parent $\mathrm{P}$ with the maximum ELT will be assigned the highest $\alpha_{p}$. Then, the illustration of workload balancing of MELT is shown in Figure 4. MELT benefits from the transmission of small packets, so it achieves high transmission performance, PDR and latency. 


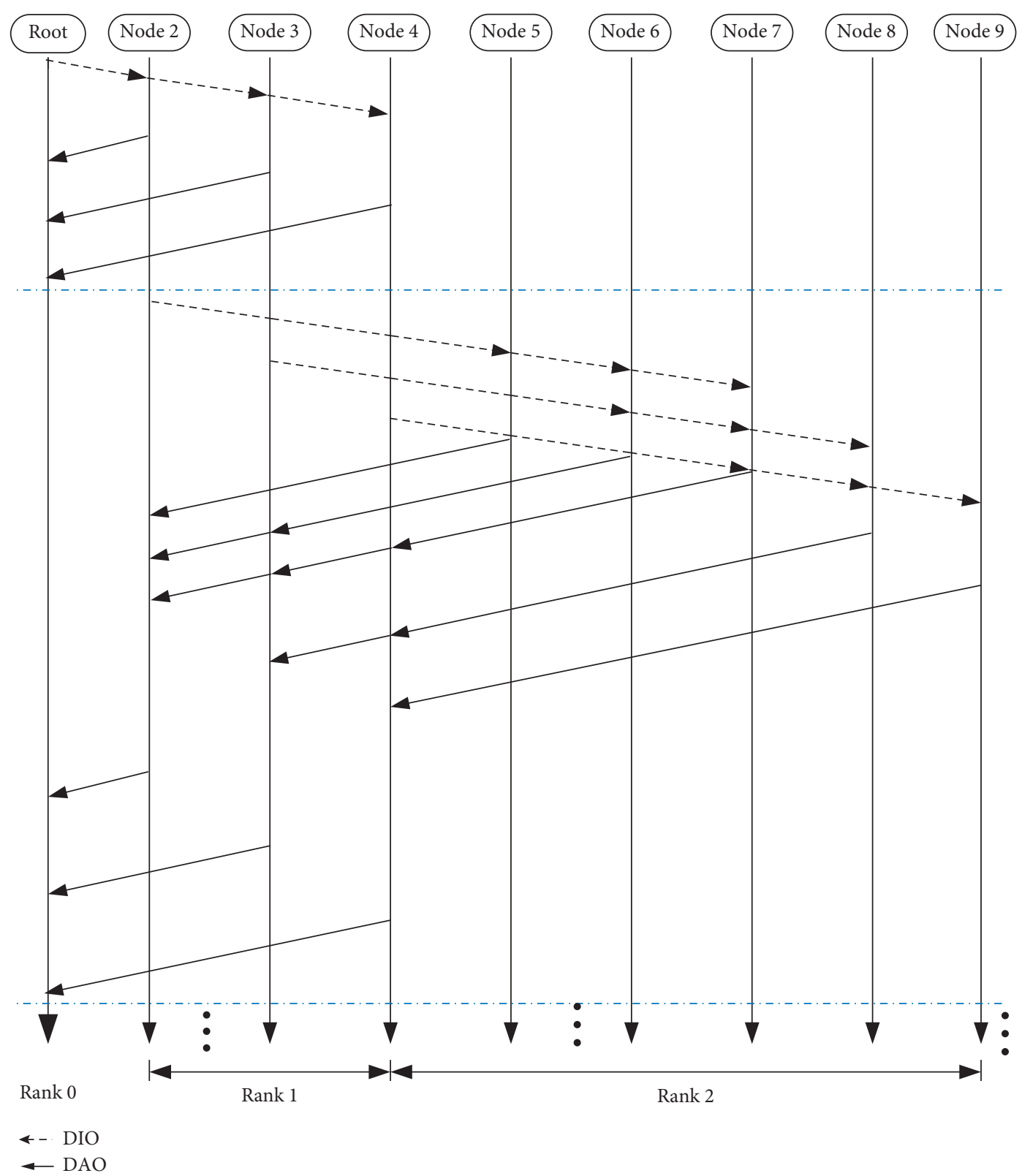

FIGURE 2: Route construction of RPL.

Moreover, MELT addresses the instability of a single parent scheme since all parents are utilized.

Multiparent selection adopts an analytic hierarchy process (MAHP) [32] to assign the split data packets to each parent based on its performance. Based on AHP algorithm, firstly the main criteria must be defined, and then each criterion undergoes a pairwise comparison according to the assigned importance weights. In MAHP, ETX, node remaining energy, and hop count $(\mathrm{HC})$ are determined as the main criteria. Then, the pairwise comparison matrix of main criteria is constructed, and the weights of main criteria are calculated using a normalized eigenvector. To generate the traffic ratio of MAHP, the values of each main criterion are determined as subcriteria, and the weights are calculated. Then, the traffic ratio is generated by determining the expected performance of the main criteria weight corresponding to subcriteria weight of each parent. By comprehensive determining of parents' quality, MAHP improves the performance of MELT. The illustration of MAHP parent selections is depicted in Figure 5.

\section{Problem Statement}

Being associated with the application of the smart grid, network lifetime, packet delivery ratio (PDR), and latency are the major concerns of low power devices, such as smart meter, of the network. The network lifetime refers to the early depletion node whose battery runs out faster than others and before the maintenance session is performed.

Once the early depletion node occurs, the root node loses the information from the early depletion node. Therefore, the DCU cannot utilize the data to perform the further 


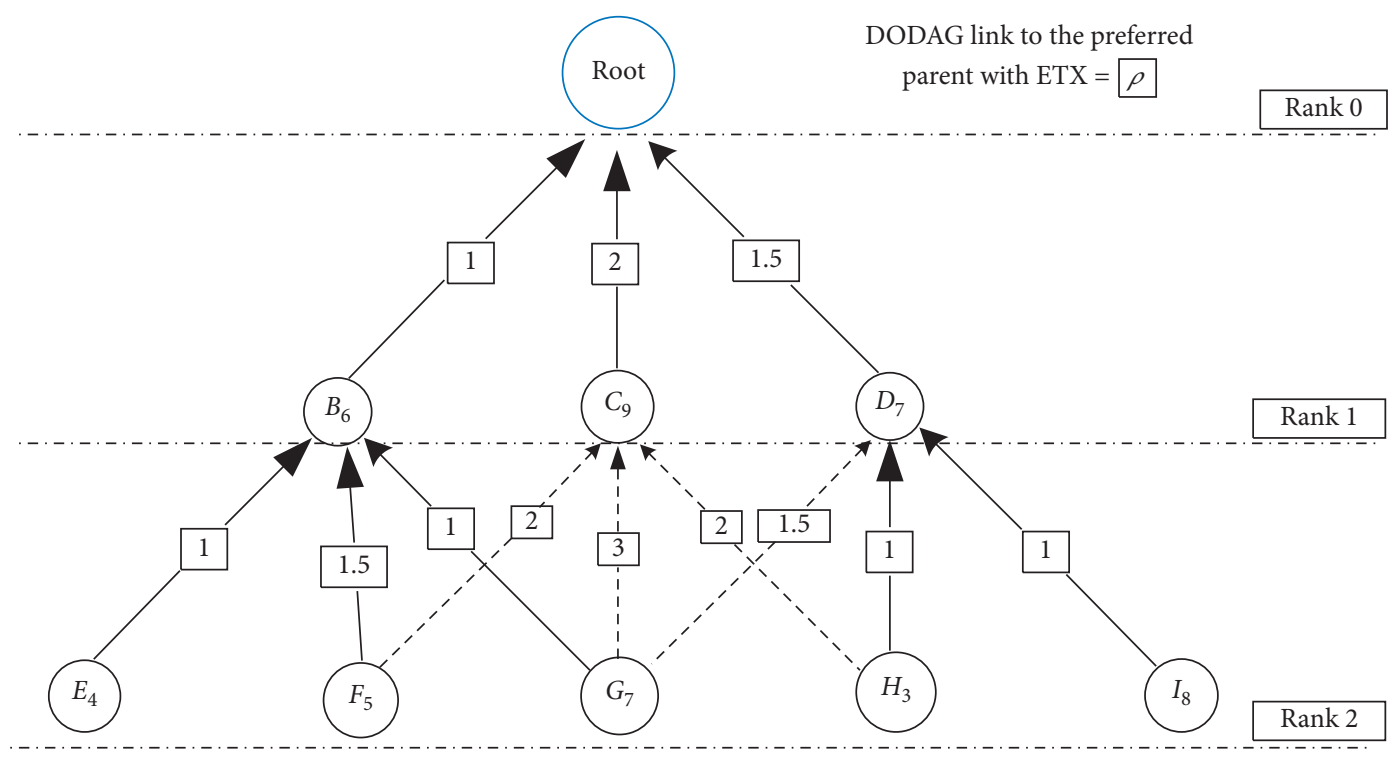

FIGURE 3: Illustration of traditional RPL parent selection.

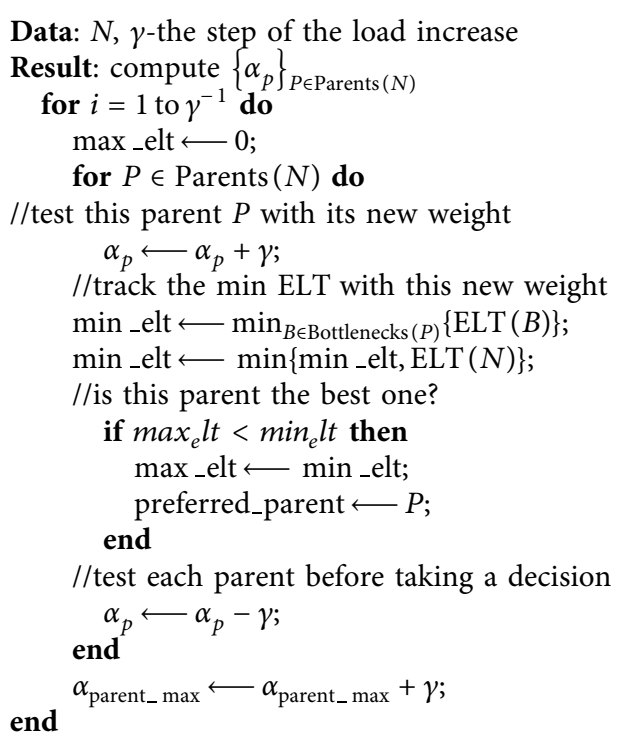

Algorithm 1: Load balancing algorithm of MELT [26].

operation. On the other hand, based on a single parent scheme, the transmission performance-PDR and latency-is affected by the quality of the immediate parent affected by the surrounding environment. Then, the parent selection function of the RPL plays an important role in avoiding the early depletion node while maintaining the reliability and latency.

3.1. Network Lifetime. Depending on the tree structure of RPL, each node is ranked according to the distance vector between the node and the root node. The node can only forward the data to the neighbors who are advertised by the different number of rank. By considering the traditional parent selection scheme with a nonconsidering workload balancing concept, the node selects the parent with the highest quality to be the immediate parent which is selected during route construction and maintenance session. Based on 3-ary tree structure, as illustrated in Figure 1, each parent has three children. For example, node 2 is considered as the parent of node 5 and node 6 , and it is considered as the candidate parent of node 7 . Node 3 is considered as the parent of node 7 , and it is considered as the candidate parent of node 6 and node 8 . Node 4 is considered as the parent of node 8 and node 9 , and it is considered as the candidate parent of node 7 .

It can be mentioned that, without taking into account the nonbalancing concept, the node only forwards its data to the best parent until the immediate parent is disabled due to battery depletion. As depicted in Figure 6(a), by considering node 2 and node 3 , it can be noticed that node 2 consumes the battery faster than node 3 because it has more children. With the passing of time, node 2 will deplete faster than node 3 due to battery depletion. Then, the local route maintenance is performed, where the DIS message is broadcasted to neighbors, as illustrated in Figure 6(b), by node 6 and it will select node 3 as the immediate parent. The dataflow of each node and the loss of information from node 2 are shown in Figure 7. Based on this scenario, the root node loses the information from node 2 since node 2 does not have the power to transmit its data. Moreover, node 5 wastes the battery usage for performing the local route maintenance request, and node 6 will have more workload since it has to forward the data from node 5 to its immediate parent. Once node 6 gains its workload, its battery will deplete faster than others and the root node will also lose the information from node 6 .

Since the workload balancing scheme is not taken into account, the depleted node will occur before the network maintenance session. Then, the utility will lose the information from the early depletion nodes. Therefore, the workload balancing scheme plays an important function for the low power device network. 


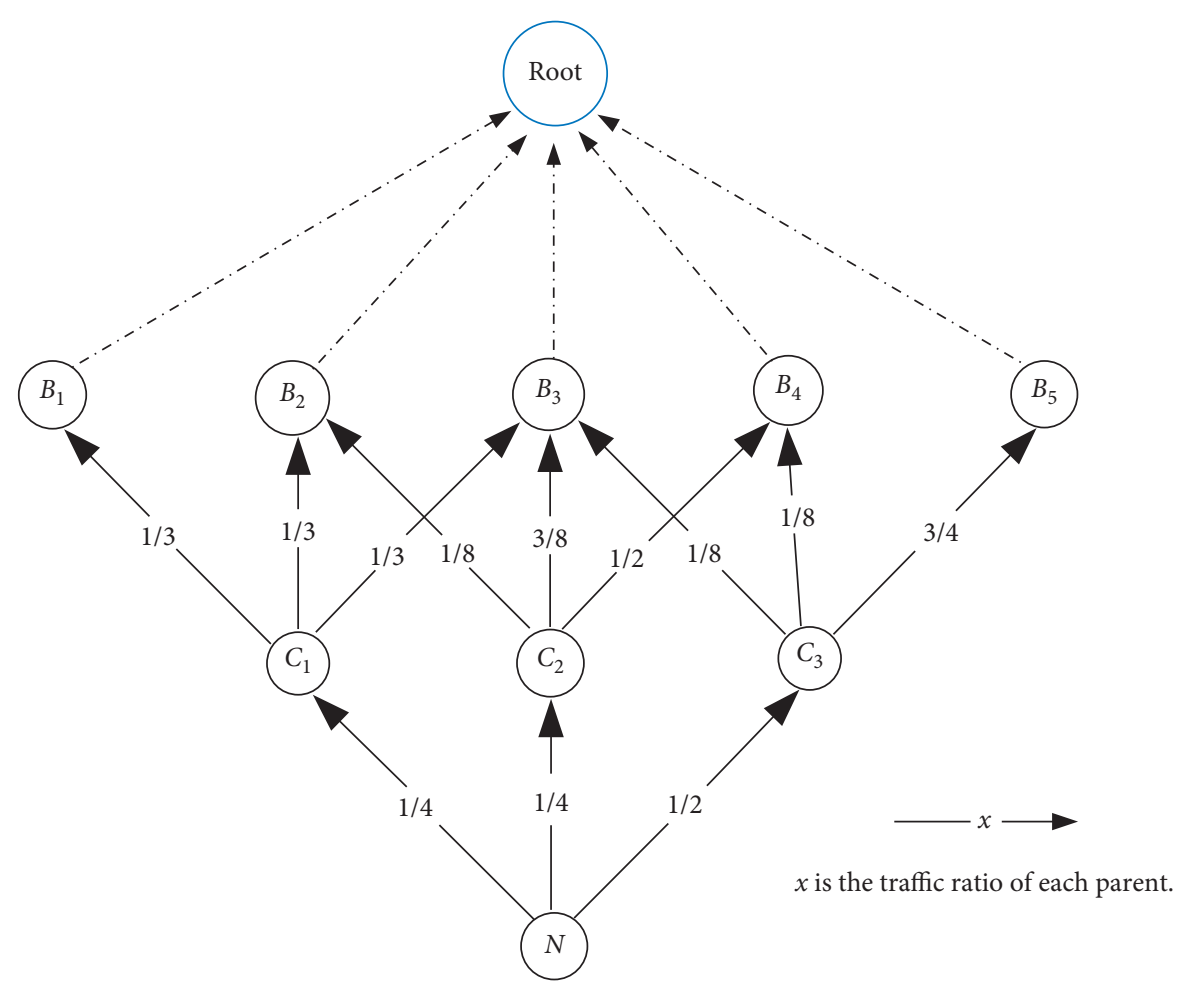

FIgURE 4: Illustration of MELT load balancing scheme.

3.2. Transmission Performance. In practice, the smart meters are considered as the static nodes; however, the quality of data transmission-PDR and latency-is affected by the surrounding environment, such as noise, distance between the child meter and the immediate parent, and data size. In general, PDR and latency are the two factors that relate to the surrounding environment directly. The environment between a child and a parent is bad; i.e., the noise is high and the child node is far from the parent. Therefore, the quality of received data by the parent will be low, and the received data will be neglected since its quality is too low to be utilized.

The packet delivery ratio (PDR) is the major factor that is used to evaluate transmission performance. PDR refers to the success transmission rate which is the ratio between the data packets transmitted by the transmitter and the packets received by the receiver. PDR should be high while latency should be low to achieve a real-time application. Generally, the transmission range and the size of data are the two major factors that degrade the PDR and increase the latency.

Once the distance between the child meter and parent is far, the transmitted package drops during the transmission and then the PDR decreases. Therefore, the retransmission is required, which increases the latency, and the child meter and parent meter must consume extra energy. This extra energy consumption refers to the energy inefficiency. On the other hand, the data package size affects transmission performance. Once the package size is high, the probability of package drop during transmission increases. Therefore, the PDR noticeably decreases on increasing package size while latency increases.
As stated by RFC standard [13], since the single parent scheme cannot meet the requirement of the transmission performance for the long transmission range and large data package size, the multiparent solution should be considered.

Although the multiparent solution presents higher transmission performance with high workload balancing than the single parent at long transmission range, a high energy consumption and low network lifetime are considered as the major demerits. Moreover, over noticeably long transmission range, the multiparent solution cannot satisfy the requirements of a high PDR and low latency due to the limited transmission channel, presents a low network lifetime, and cannot achieve high energy efficiency.

Therefore, in this paper, we proposed a hybrid solution that adapts the parameter to transmit the data packet between the multiparent solution and the single parent with cognitive radio based on the expected performance determined by the advanced multicriteria ranking method.

\section{Empowered Hybrid Parent Selection}

In this paper, we propose the empowered hybrid parent selection (EHPS) algorithm to maximize the network lifetime and achieve a high packet delivery ratio (PDR) and low latency using hybridizing scheme of the multiparent solution and the single parent with cognitive radio. The fuzzy analytic hierarchy process (FAHP) [33-35] is exploited where the expected transmission count (ETX), expected lifetime (ELT), and expected transmission time (ETT) [22] are determined as the main criteria, to analyze the quality of 


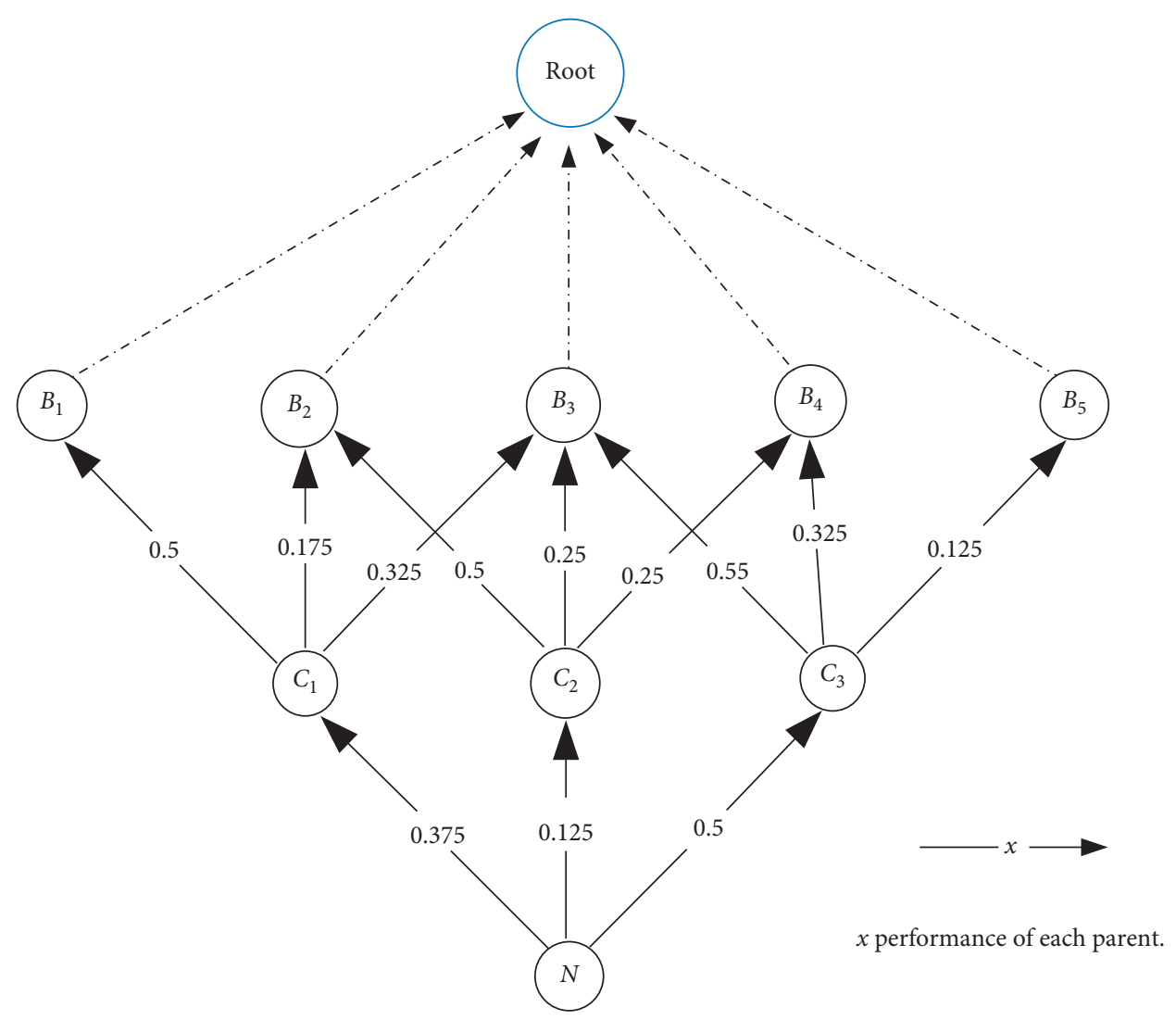

FIgURE 5: Illustration of MAHP load balancing scheme.

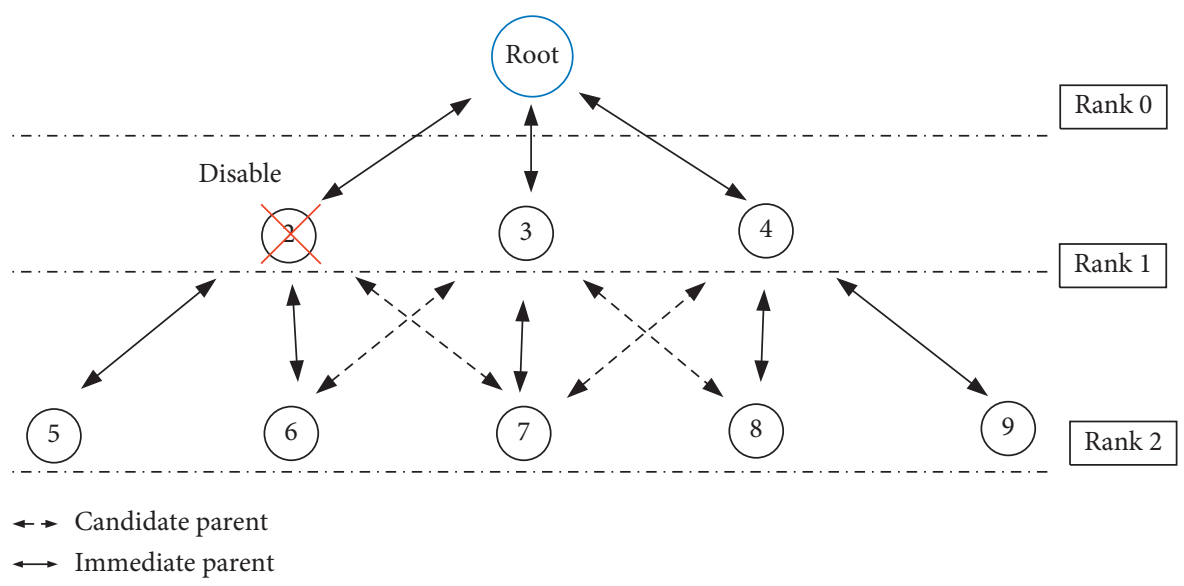

(a)

FIGURE 6: Continued. 


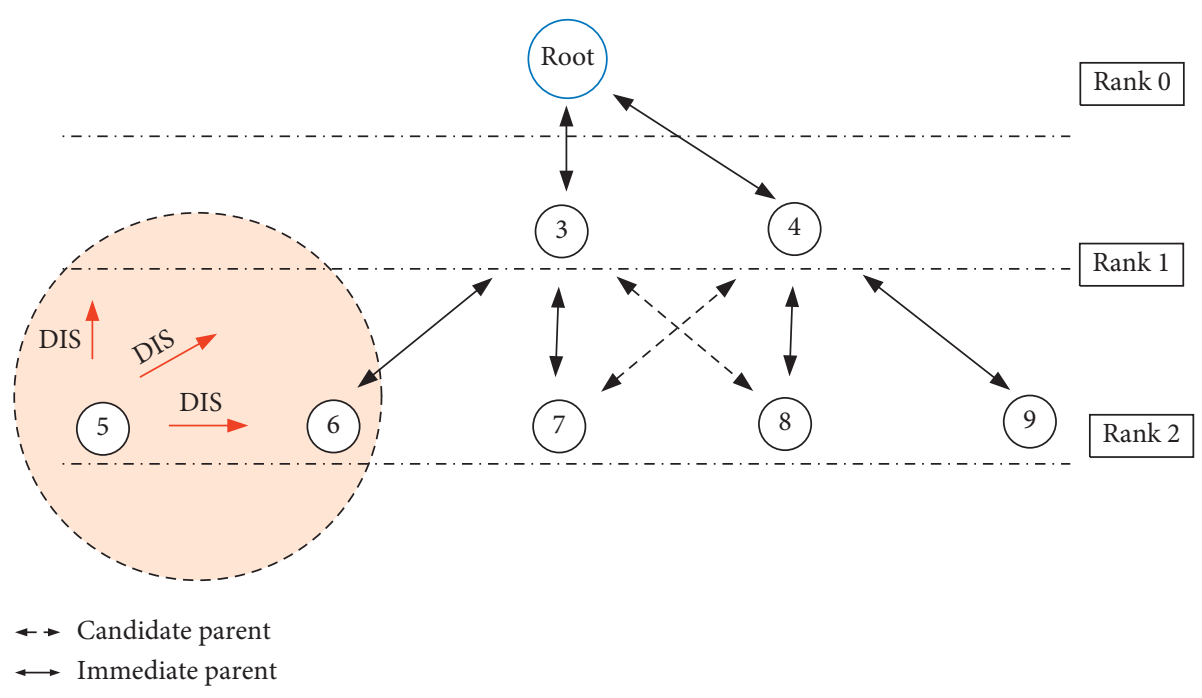

(b)

Figure 6: Occurrence of early depletion node: (a) 3-ary tree structure of RPL network with node 2 disabled; (b) local route maintenance session performed by node 5 .

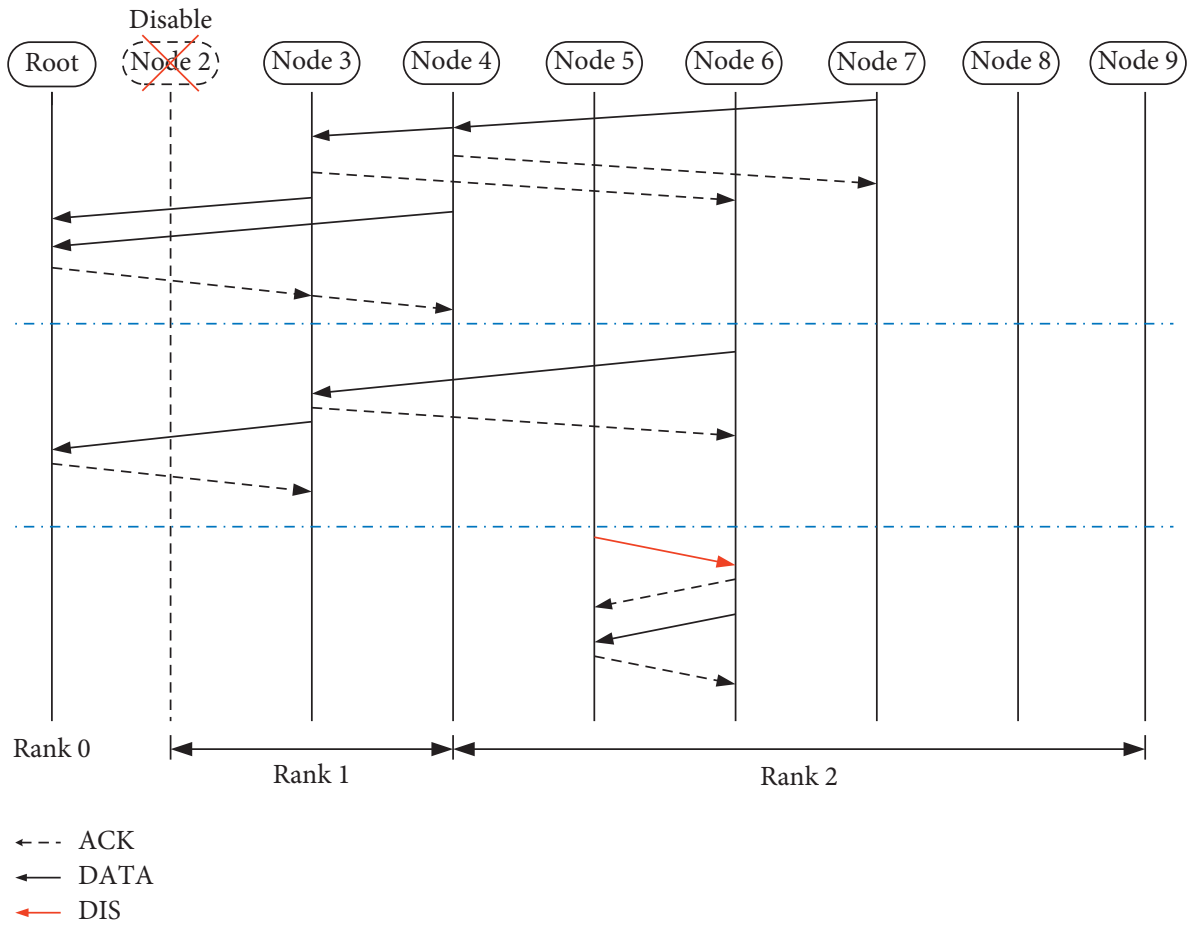

FIGURE 7: Framework of data transmission when node 2 is disabled.

parent meters comprehensively. In FAHP, the fuzzy set is exploited with the traditional AHP to address the unbalance judgment scaling of traditional version. To achieve high network lifetime, the workload balancing capability is improved by using influence weight adapting scheme. From our study, the energy consumption increases on decreasing meter's ranking in an exponential manner. Therefore, our algorithm adapts the influence of energy efficiency through ELT criteria exponentially. Moreover, the single parent with cognitive radio is adopted in a hybridizing scheme with multiparent solution to achieve a high PDR rate, high energy efficiency, and low latency under a long transmission range.

The framework of EHPS is shown in Figure 8 which can be categorized into offline and online mode. In an offline mode, once the algorithm is activated, the three main criteria-ETX, ELT, and ETT-are pairwise compared 


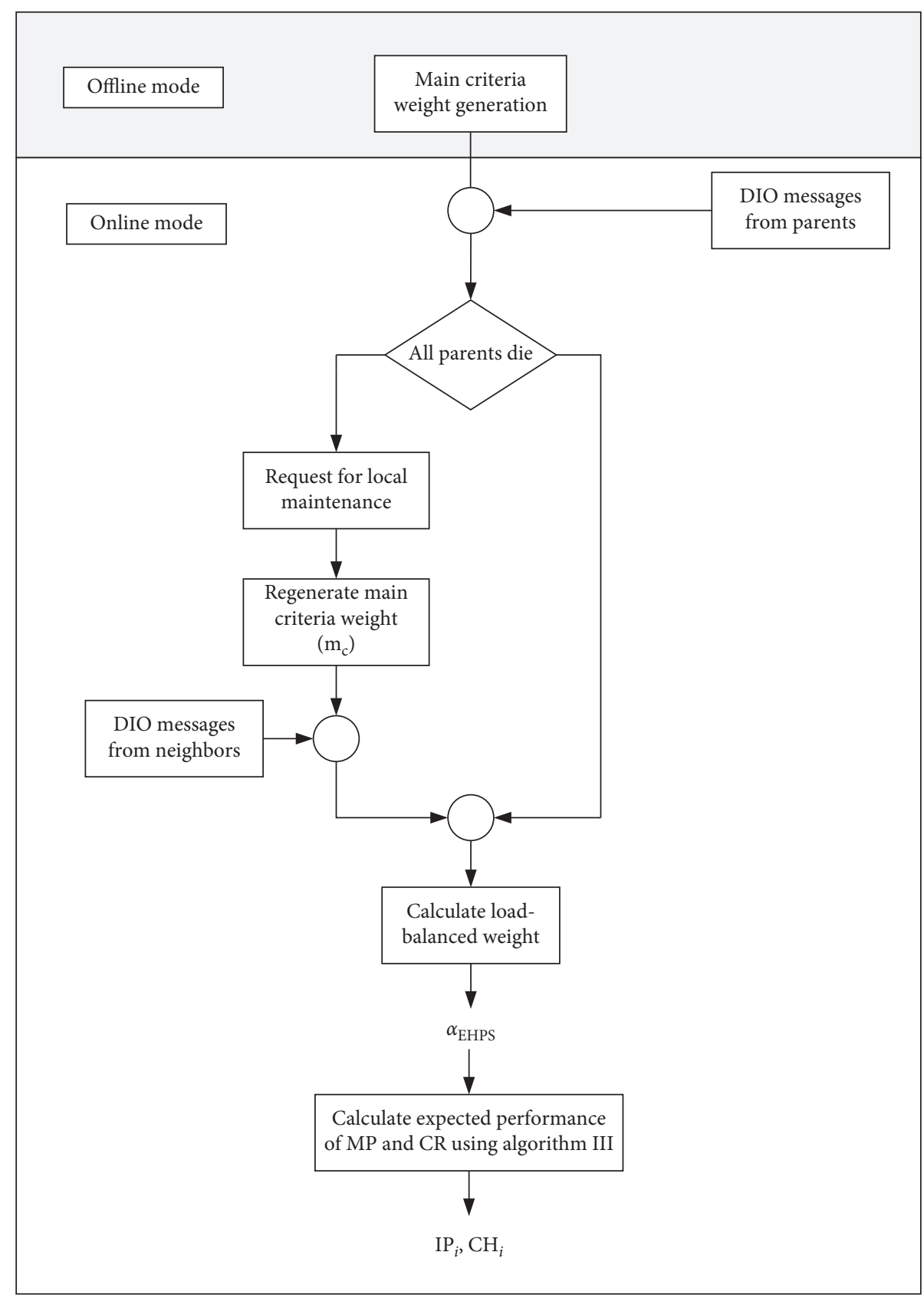

FIGURE 8: Framework of EHPS.

according to FAHP theorem to generate the main criteria weight $\left(\mathbf{m}_{c}\right)$. Depending on tree topology, the level of energy consumption in each rank is different according to the number of children; then, the requirement of workload balancing in each rank should be different. Therefore, in our algorithm, we determine the effect of network ranking as the factor for performing a pairwise comparison in multicriteria ranking process. Then, the pairwise comparison between ETX and ELT is varied based on the number of rank; e.g., ELT factor in rank 4 should be more important than ELT factor in rank 5 because a parent in rank 4 has a higher number of children than a parent in rank 5 .
In an online mode, firstly, the node receives the DIO messages from a number of candidate parents, where the message contains its ELT. Secondly, the node performs the ELT calculation of those candidate parents by adding its traffic to determine the actual ELT when the traffic of the node is taken into account. If all candidate parents die, the node will broadcast DIS message to request the local maintenance process. Once the neighbors receive the DIS message, they will transmit DIO message to the node. Then, the node will calculate the load-balanced weight through FAHP procedure.

To calculate ELT, firstly, the data traffic from the node to be transmitted to the parents is determined, where the data traffic $(T)$ is given by 


$$
T=T_{\text {node }}+\sum_{i \in \text { Children(node) }} T_{i} .
$$

Secondly, the energy consumption $(E)$ of the node included with the retransmission factor is calculated, which can be expressed as

$$
E(N)=\frac{T \times\left(\sum_{i \in \text { Children (node) }} \alpha_{i}\right) \times \operatorname{ETX}(\text { node }, C)}{\text { Data_rate }} \times P_{\mathrm{TX}},
$$

where $\alpha_{i}$ is the traffic ratio in which the children forward the packets to the node, $P_{T X}$ is the transmission power, and Data_rate is the data transmission rate.

Finally, the expected lifetime (ELT) of node $N$ is given as

$$
\operatorname{ELT}(N)=\frac{E_{\text {res }}(N)}{E(N)} \text {. }
$$

By exploiting FAHP theorem [35], firstly, pairwise comparison is made on each main criterion; therefore, weight of importance between criteria should be assigned. As mentioned earlier, the effect of meter's ranking where the energy consumption changes exponentially is taken into account, and it is determined as the multiplication factor ( $\mathrm{mf}$ ) to be used in the pairwise comparison process. Then, the $\mathrm{mf}$ can be derived as follows. The exponential change of energy consumption can be expressed as

$$
y=C e^{b x}
$$

where $C$ is the constant value, $b$ is the growth rate of exponential function, and energy consumption is considered as the input data $(x)$.

Then, the decaying exponential function can be rewritten as

$$
\ln y=\ln C+b x
$$

Therefore, the fitting values can be given as

$$
b=\frac{n \sum_{i=1}^{n} x_{i} \ln y_{i}-\sum_{i=1}^{n} x_{i} \sum_{i=1}^{n} \ln y_{i}}{n \sum_{i=1}^{n} x_{i}^{2}-\left(\sum_{i=1}^{n} x_{i}\right)^{2}}
$$

where $n$ is the amount of data.

In our algorithm, we utilize the exponential function with the growth rate where influence of rank is taken into account. Therefore, the multiplication factor $(\mathrm{mf})$ is given as

$$
\mathrm{mf}=e^{b\left(\text { Rank }_{\max }-\text { Rank }_{\text {current }}\right)},
$$

where Rank $_{\text {max }}$ is the maximum rank of the network and Rank $_{\text {current }}$ is the current rank.

Therefore, to determine the load-balanced weight of EHPS $\left(\alpha_{\mathrm{EHPS}}=\left[\alpha_{\mathrm{EHPS}_{1}}, \alpha_{\mathrm{EHPS}_{2}}, \ldots, \alpha_{\mathrm{EHPS}_{k}}\right]\right)$, the structure of multicriteria ranking according to FAHP algorithm is constructed as depicted in Figure 9. In our proposed algorithm, ETX, ELT, and ETT are determined as the main criteria. The actual values of each main criterion are determined as the input of subcriteria.

The framework of EHPS is illustrated in Algorithm 2. Once the objective, main criteria, and subcriteria are defined, firstly, each main criterion is pairwise compared to others by constructing the $M \times M$ of pairwise comparison matrix (A), where $M$ is the number of main criteria. As mentioned earlier, by taking $\mathrm{mf}$ into account, the pairwise comparison matrix (A) can be given by

$$
\mathbf{A}=\left[\begin{array}{cccc}
\frac{1}{\mathrm{mf}} a_{21} & 1 & a_{23} \\
a_{31} & a_{32} & 1
\end{array}\right],
$$

where $a_{12}$ represents the weight of importance between ETX and ELT, $a_{13}$ represents the weight of importance between ETX and ETT, and $a_{23}$ represents the weight of importance between ELT and ETT. In general, $a_{j i}$ is the comparison of importance between criteria where $a_{j i}$ is equal to $1 / a_{i j}$. The scale of importance comparison is in the range of 9 to $1 / 9$. For example, if $a_{12}$ is 1, ETX is as important as ELT. By taking the multiplication factor ( $\mathrm{mf}$ ) into account, the importance of ETX is changed based on the effect of rank level.

Secondly, the fuzzy comparison matrix $(\widetilde{A})$ is generated according to the pairwise comparison matrix (A) with respect to the fuzzy triangular number which is shown in Table 1.

$$
\widetilde{A}=\left[\begin{array}{ccc}
\tilde{d}_{11} & \cdots & \tilde{d}_{1 n} \\
\vdots & \ddots & \vdots \\
\tilde{d}_{n 1} & \cdots & \tilde{d}_{n n}
\end{array}\right],
$$

where $n$ is three times the number of the main criteria which is based on the fuzzy rule.

Thirdly, the fuzzy comparison matrix $(\widetilde{A})$ performs geometric means calculation $\left(\widetilde{r}_{i}\right)$ which can be given as

$$
\widetilde{r}_{i}=\left(\prod_{j=1}^{n} \tilde{d}_{i j}\right)^{1 / n}, \quad i=1,2, \ldots, n .
$$

Fourthly, the fuzzy weights are generated by performing the vector summation of each $\widetilde{r}_{i}$ and inversing each summation vector. Therefore, the maximum order $\left(u w_{i}\right)$, average order $\left(m w_{i}\right)$, and minimum order $\left(l w_{i}\right)$ are obtained. Then, the fuzzy weights $\left(\widetilde{w}_{i}\right)$ can be obtained by

$$
\widetilde{w}_{i}=\widetilde{r}_{i} \otimes\left(\widetilde{r}_{1} \oplus \widetilde{r}_{2} \oplus \cdots \oplus \widetilde{r}_{n}\right)^{-1},
$$

where $\oplus$ is a direct sum and $\otimes$ is a dyadic product.

Then, the fuzzy weights are defuzzified by

$$
\mathrm{d} w_{i}=\frac{l w_{i}+m w_{i}+u w_{i}}{3} .
$$

Finally, the main criteria weight $\left(\mathbf{m}_{\mathbf{c}}=\left[m_{c_{1}}, m_{c_{2}}, \ldots, m_{c_{M}}\right]\right)$ is generated by normalizing the defuzzified vector (dw) when the normalized defuzzified vector $(\mathbf{d w})$ can be expressed as

$$
m_{c_{i}}=\frac{\mathrm{d} w_{i}}{\sum_{i=1}^{M} \mathrm{~d} w_{i}}
$$

After the main criteria weight is calculated, the subcriteria weights $\left(s_{c}^{C}=\left[s_{c_{1}}^{C}, s_{c_{2}}^{C}, \ldots, s_{c_{m}}^{C}\right]\right)$ of each main 


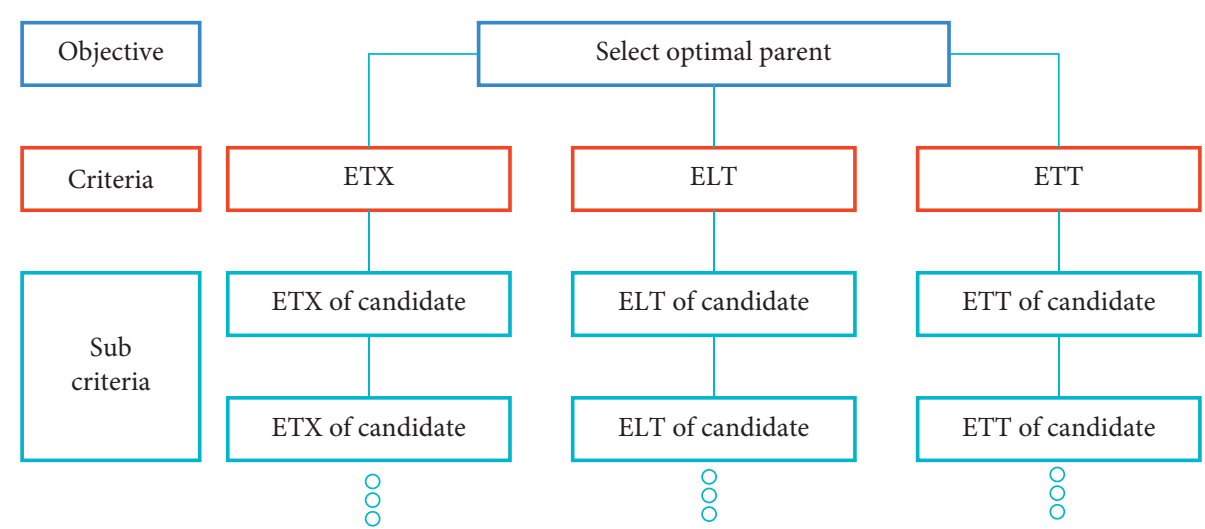

FIGURE 9: Structure of multicriteria ranking according to FAHP algorithm.

Input $k$, ELT, ETX and ETT

Output Load-balanced weight $\left\{\alpha_{\text {EHPS }}\right\}$

if All candidate parents die then

Broadcast DIS message to perform local maintenance;

Increase the rank number of the node;

end

Calculate the multiplication factor ( $\mathrm{mf}$ ) according to the rank;

Calculate weight of main criteria $\left\{m_{c}\right\}$ according to $\mathrm{mf}$;

Calculate weight of subcriteria $\left\{s_{c}^{C}\right\}$; for $i \in k$

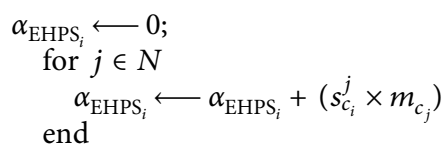

end

Generate $\alpha_{\mathrm{EHPS}}$ by calculating $s_{c}$ according to $\mathbf{m}_{c}$;

Algorithm 2: Multicriteria load balancing algorithm.

TABLE 1: Interpretation of the significance of influence in triangular fuzzy scale [35].

\begin{tabular}{lcc}
\hline Influence in a numeric scale & Definition & Triangular fuzzy scale \\
\hline 1 & Equal significance & $(1,1,1)$ \\
3 & Moderate significance & $(2,3,4)$ \\
5 & Strong significance & $(4,5,6)$ \\
7 & Very strong significance & $(6,7,8)$ \\
9 & Extreme significance & $(9,9,9)$ \\
2 & & $(1,2,3)$ \\
4 & & $(3,4,5)$ \\
6 & Intermediate values between two adjacent judgments & $(5,6,7)$ \\
8 & & $(7,8,9)$ \\
\hline
\end{tabular}

criterion are calculated as the main criteria procedures. Therefore, the load-balanced weight $\left(\alpha_{\mathrm{EHPS}}=\left[\alpha_{\mathrm{EHPS}_{1}}, \alpha_{\mathrm{EHPS}_{2}}, \ldots, \alpha_{\mathrm{EHPS}_{k}}\right]\right)$ is generated by computing $s_{c}$ according to $\mathbf{m}_{\mathbf{c}}$.

It should be mentioned that the load-balanced weight of EHPS $\left(\alpha_{\text {EHPS }}\right)$ can be expressed the quality of each parent. Then, we select the lowest quality parent and estimate its performance according to the cognitive radio environment. Then, the expected quality of the selected parent on cognitive radio is $\mathrm{ELT}_{\mathrm{CR}}, \mathrm{ETX}_{\mathrm{CR}}$, and $\mathrm{ETT}_{\mathrm{CR}}$. To compare the performance of multiparent solution and the single parent with cognitive radio, the expected quality of the multiparent solution-ELT $\mathrm{EP}_{\mathrm{MP}}, \mathrm{ETX}_{\mathrm{MP}}$, and ETT $_{\text {MP }}$-is calculated according to $\alpha_{\text {EHPS }}$. Therefore, the expected quality of the selected parent on cognitive radio and multiparent solution is considered as the input of hybrid parent selection algorithm and used as the subcriteria. 
As shown in Algorithm 3, the main criteria weight from multicriteria load balancing algorithm is used as the main criteria weight of hybrid parent selection. Then, subcriteria weights $\left(s_{h}^{C}=\left[s_{h_{\mathrm{MP}}^{C}}^{C}, s_{h_{\mathrm{CR}}}^{C}\right]\right)$ are calculated, where $s_{h_{\mathrm{MP}}^{C}}^{C}$ is the subcriteria weight of multiparent solution and $s_{h_{\mathrm{CR}}}^{\mathrm{MP}^{\mathrm{P}}}$ is the subcriteria weight of single parent with cognitive radio. Therefore, the performance weight $\left(\mathbf{P e r}=\left[\mathrm{Per}_{1}, \mathrm{Per}_{2}\right]\right)$ is calculated, where $\mathrm{Per}_{1}$ is the expected performance of multiparent solution and $\mathrm{Per}_{2}$ is the expected performance of selected parent on cognitive radio. If the performance of the multiparent solution is higher than the single parent with cognitive radio, then the multiparent solution is activated and the child meter forwards the data to multiple parents according to $\alpha \mathbf{r}_{\text {EHPS }}$. Otherwise, the child meter transmits the DIS message to the selected parent to inform it about changing of communication channel to the cognitive radio. The output of Algorithm 3 presents the selected parent and the transmission technology as shown in Table 2.

\section{Simulation Results}

In this section, we simulate the performance of the empowered hybrid parent selection (EHPS) algorithm under 3-ary tree topology of AMI network. The performance of EHPS is evaluated as compared to 3 former parent selection methods: traditional RPL, multiparent selection based on expected lifetime (MELT) [26], and multiparent selection based on an analytic hierarchy process (MAHP) [32]. The three critical network performance metrics, packet delivery ratio (PDR), latency, and network lifetime, are determined. The PDR and latency are used to determine the transmission performance where PDR is the ratio of the packets transmitted by the child meter to the packets received by the parent meter. Latency is defined as the data transmission time. As stated in [14], the required PDR, data reliability, for transmitting the energy usage information is as high as 0.98 .

5.1. Simulation Setup. To simulate the performance of parent selection, based on 3-ary tree topology, the quality of the middle parent is set to be the best where the distance of the middle parent to the child is set to be less than that of other parents. For example, as depicted in Figure 1, node 3 is considered as the middle parent. Therefore, the distance of node 3 to node 7 is less than the distance of node 2 and node 4 to node 7 . To investigate the effect of path loss, the distance between the middle parent and the child ranges between 10 and 80 meters. The specification of transmitter and receiver is based on Zigbee module CC2500 [38]. All simulations are performed on MATLAB. Other parameters are shown in Table 3.

As described earlier, to generate the load-balanced weight $\left(\alpha_{\text {EHPS }}\right)$ of EHPS, firstly the pairwise comparison between performance metrics is performed. In this paper, we set the influence of each metric equally and adapt the influence of energy efficiency through the multiplication factor ( $\mathrm{mf})$. The $\mathrm{mf}$ is gathered by using (11), where $b$ is estimated from the meter lifetime when the workload of multiparent is perfectly balanced; i.e., all parents share data packets equally. Then, $b$ is set to -0.5443 . Therefore, the pairwise comparison matrix (A) is given as

$$
\mathbf{A}=\left[\begin{array}{ccc}
1 & \mathrm{mf} & 1 \\
\frac{1}{\mathrm{mf}} & 1 & 1 \\
1 & 1 & 1
\end{array}\right]
$$

where

$$
\mathrm{mf}=e^{-0.5443\left(\text { Rank }_{\max }-\text { Rank }_{\text {current }}\right)}
$$

5.2. Performance Evaluation. Firstly, we determine the network lifetime presented by each parent selection algorithm as a function of the rank number and the distance between child meter and parents. As depicted in Table 4, for a single parent based on RPL algorithm, the number of rank affects the decrease in network lifetime noticeably since the number of forwarded packets by the parent node is corresponding to the rank number. The lower the rank advertised by a node is, the higher the number of data packets from the children and grandchildren meters is determined. On the other hand, the transmission range also affects the network lifetime. If the distance between a meter and parent meter increases, the times of required data retransmission increase due to the packet loss. Therefore, for RPL algorithm, the network lifetime decreases when the distance is higher than $50 \mathrm{~m}$. When the distance is greater than $65 \mathrm{~m}$, the meter wastes the energy for retransmission, so the network lifetime is noticeably low.

As depicted in Tables 5 and 6, the network lifetimes of MELT and MAHP are determined, respectively. Although the multiparent solutions have the merits of efficient workload balancing and high PDR, they also have the demerit of high energy consumption since the information needs to be split and forwarded to a number of parents. On the contrary, as shown in Table 7, EHPS algorithm considers the quality of parents comprehensively through a fuzzy AHP (FAHP) algorithm and through activating the cognitive radio technology on the single parent scheme; therefore, it overcomes the tradeoff between high energy consumption and high transmission performance of multiparent solution effectively. Therefore, the EHPS can maximize the network lifetime under long transmission range environment.

Consequently, RPL algorithm presents the highest network lifetime when the distance is lower than $50 \mathrm{~m}$. It should be noted that retransmission is not performed at those distances since the PDR is as high as 1 . When the distance increases, the data retransmission is required; therefore, the network lifetime for RPL is noticeably low. Although, the multiparent solutions-MELT and MAHP_-present better workload balancing performance by activating all candidate parents when the data transmission is performed, they present a lower network lifetime than the RPL algorithm since they consume more energy in transmitting a number 


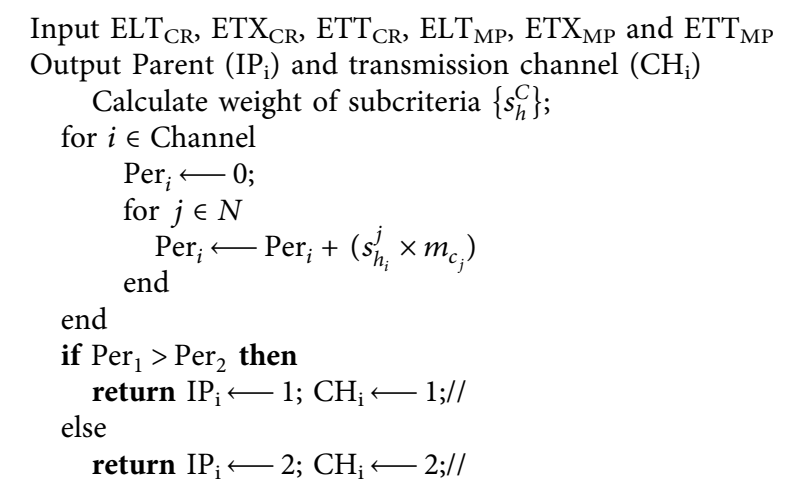

Algorithm 3: Hybrid parent selection algorithm.

TABLE 2: Interpretation of the outputs of Algorithm 3.

\begin{tabular}{lcc}
\hline $\begin{array}{l}\text { Subscripted } \\
\text { number (i) }\end{array}$ & 1 & 2 \\
\hline $\mathrm{CH}_{\mathrm{i}}$ & Zigbee & $\mathrm{CR}$ \\
$\mathrm{IP}_{\mathrm{i}}$ & All candidate parents & Selected single parent \\
\hline
\end{tabular}

TABLE 3: Simulated parameters [38].

\begin{tabular}{lc}
\hline Parameters & Value \\
\hline Tx power & $76.3 \mathrm{~mW}$ \\
CR Tx power & $100 \mathrm{~mW}$ \\
Rx power & $47.9 \mathrm{~mW}$ \\
Idle power & $900 \mathrm{nW}$ \\
Sleep power & $400 \mathrm{nW}$ \\
Spectrum sensing power & $65.83 \mathrm{~mW}$ \\
DIO interval before data transmitting & 90 seconds \\
Household data transmitting interval & $15 \mathrm{~min}$ \\
Manufacturer data transmitting interval & $60 \mathrm{~min}$ \\
Household data packet & 318 bytes \\
Manufacturer data packet & $1200 \mathrm{bytes}$ \\
DIO message size & 64 bytes \\
DAO message size & $46 \mathrm{bytes}$ \\
DIS message size & 2 bytes \\
Transmission time of data packet & $192 \mathrm{us}$ \\
Transition time from sleep to active mode & $970 \mathrm{us}$ \\
Simulation time & $3600 \mathrm{~min}$ \\
Load balancing step of MELT & $1 / 3$ \\
\hline
\end{tabular}

of small packets to multiple parents. On the other hand, EHPS presents a network lifetime close to that of the RPL algorithm when the distance is lower than $50 \mathrm{~m}$ by hybridizing the multiparent solution and the single parent with cognitive radio technology. Moreover, with the merit of cognitive radio, EHPS presents the highest network lifetime when the transmission range is greater than $50 \mathrm{~m}$.

Secondly, we evaluate the PDR and latency of EHPS as compared to RPL, MELT, and MAHP as a function of distance at rank 9 during household data transmission. As shown in Figure 10, the comparison of PDR is determined. As a result, all algorithms present PDR as high as 1 when the distance between meter and parent meters is lower than $50 \mathrm{~m}$. When the distance increases, the PDR of the RPL algorithm is the worst since its performance suffers from packet loss according to the package size and distance. On the other hand, MELT, MAHP, and EHPS present higher PDR since the data is split into small packets based on the multiparent solution. Moreover, by our proposed cognitive radio hybridizing scheme, EHPS presents the highest PDR among others since it utilizes better transmission channel opportunistically. The single parent based on cognitive radio is activated by the performance comparison to multiparent solution through the proposed hybrid parent selection algorithm.

As shown in Figure 11, the latency values of RPL, MELT, MAHP, and EHPS are compared. The simulation results show that EHPS presents the lowest latency as compared to others. By utilizing the cognitive radio continually, EHPS can present efficient data transmission performance, high PDR and low latency, while maximizing the network lifetime since the quality of parents is determined comprehensively. Moreover, by adapting the influence of energy-efficient criteria on the rank number, the workload can be balanced effectively.

Thirdly, we evaluate the PDR and latency of EHPS as compared to RPL, MELT, and MAHP as a function of distance at rank 9 during manufacturer data transmission. It should be mentioned that the interval of the energy usage of manufacturer data transmission is $60 \mathrm{~min}$, and the data package size is 1200 bytes which is much higher than the size of household energy usage. As mentioned earlier, the data package size affects the PDR, latency, and required retransmission. As shown in Figure 12, the PDR of the RPL algorithm decreases when the distance is $45 \mathrm{~m}$. Its PDR noticeably decreases when the distance increases and reaches 0 at $80 \mathrm{~m}$. Although MELT and MAHP present much better PDRs than the RPL, their PDRs are also noticeably low on increasing distance due to the limited communication channel. In addition, the PDRs of MELT and MAHP are 0 at $80 \mathrm{~m}$, the same as RPL algorithm.

As a result, EHPS overcomes the limited performance of Zigbee communication channel by hybridizing the multiparent solution and the single parent with cognitive radio 
TABLE 4: Network lifetime of RPL.

\begin{tabular}{|c|c|c|c|c|c|c|c|c|}
\hline \multirow{2}{*}{ Distance $(\mathrm{m})$} & \multicolumn{8}{|c|}{ Rank } \\
\hline & 2 & 3 & 4 & 5 & 6 & 7 & 8 & 9 \\
\hline 10 & 8245 & 4620 & 2915 & 1990 & 1425 & 1100 & 860 & 695 \\
\hline 15 & 8245 & 4620 & 2915 & 1990 & 1425 & 1100 & 860 & 695 \\
\hline 20 & 8245 & 4620 & 2915 & 1990 & 1425 & 1100 & 860 & 695 \\
\hline 25 & 8245 & 4620 & 2915 & 1990 & 1425 & 1100 & 860 & 695 \\
\hline 30 & 8245 & 4620 & 2915 & 1990 & 1425 & 1100 & 860 & 695 \\
\hline 35 & 8245 & 4620 & 2915 & 1990 & 1425 & 1100 & 860 & 695 \\
\hline 40 & 8245 & 4620 & 2915 & 1990 & 1425 & 1100 & 860 & 695 \\
\hline 45 & 8240 & 4620 & 2915 & 1990 & 1425 & 1100 & 860 & 695 \\
\hline 50 & 8225 & 4605 & 2905 & 1985 & 1425 & 1095 & 860 & 695 \\
\hline 55 & 8120 & 4540 & 2865 & 1960 & 1425 & 1080 & 845 & 680 \\
\hline 60 & 7655 & 4230 & 2650 & 1805 & 1320 & 995 & 785 & 630 \\
\hline 65 & 6170 & 3305 & 2040 & 1385 & 995 & 750 & 590 & 475 \\
\hline 70 & 2525 & 1265 & 725 & 480 & 310 & 245 & 185 & 140 \\
\hline 75 & 125 & 65 & 65 & 65 & 65 & 65 & 65 & 65 \\
\hline 80 & 65 & 65 & 65 & 65 & 50 & 40 & 30 & 25 \\
\hline
\end{tabular}

TABle 5: Network lifetime of MELT.

\begin{tabular}{|c|c|c|c|c|c|c|c|c|}
\hline \multirow{2}{*}{ Distance $(\mathrm{m})$} & \multicolumn{8}{|c|}{ Rank } \\
\hline & 2 & 3 & 4 & 5 & 6 & 7 & 8 & 9 \\
\hline 10 & 6930 & 3725 & 2230 & 1485 & 1060 & 795 & 630 & 515 \\
\hline 15 & 6930 & 3725 & 2230 & 1485 & 1060 & 795 & 630 & 515 \\
\hline 20 & 6930 & 3725 & 2230 & 1485 & 1060 & 795 & 630 & 515 \\
\hline 25 & 6930 & 3725 & 2230 & 1485 & 1060 & 795 & 630 & 515 \\
\hline 30 & 6930 & 3545 & 2100 & 1385 & 975 & 725 & 570 & 450 \\
\hline 35 & 6930 & 3545 & 2100 & 1385 & 975 & 725 & 570 & 450 \\
\hline 40 & 6930 & 3545 & 2100 & 1385 & 975 & 725 & 570 & 450 \\
\hline 45 & 6930 & 3545 & 2100 & 1385 & 975 & 725 & 570 & 450 \\
\hline 50 & 6920 & 3535 & 2095 & 1385 & 975 & 725 & 565 & 450 \\
\hline 55 & 6845 & 3485 & 2065 & 1365 & 965 & 720 & 555 & 440 \\
\hline 60 & 6545 & 3305 & 1955 & 1285 & 905 & 665 & 530 & 420 \\
\hline 65 & 5405 & 2645 & 1555 & 1005 & 705 & 535 & 400 & 305 \\
\hline 70 & 1545 & 905 & 545 & 305 & 215 & 185 & 125 & 95 \\
\hline 75 & 65 & 65 & 65 & 65 & 65 & 65 & 65 & 65 \\
\hline 80 & 65 & 65 & 65 & 65 & 55 & 40 & 30 & 25 \\
\hline
\end{tabular}

TABle 6: Network lifetime of MAHP.

\begin{tabular}{|c|c|c|c|c|c|c|c|c|}
\hline \multirow{2}{*}{ Distance $(\mathrm{m})$} & \multicolumn{8}{|c|}{ Rank } \\
\hline & 2 & 3 & 4 & 5 & 6 & 7 & 8 & 9 \\
\hline 10 & 7760 & 4240 & 2610 & 1750 & 1255 & 935 & 725 & 580 \\
\hline 15 & 7760 & 4240 & 2610 & 1750 & 1255 & 935 & 725 & 580 \\
\hline 20 & 7760 & 4240 & 2610 & 1750 & 1255 & 935 & 725 & 580 \\
\hline 25 & 7760 & 4240 & 2610 & 1750 & 1255 & 935 & 725 & 580 \\
\hline 30 & 7770 & 4250 & 2610 & 1750 & 1255 & 935 & 725 & 580 \\
\hline 35 & 7770 & 4250 & 2610 & 1750 & 1255 & 935 & 725 & 580 \\
\hline 40 & 7770 & 4250 & 2610 & 1750 & 1255 & 935 & 725 & 580 \\
\hline 45 & 7770 & 4250 & 2610 & 1745 & 1255 & 935 & 725 & 580 \\
\hline 50 & 7745 & 4240 & 2610 & 1720 & 1255 & 935 & 725 & 580 \\
\hline 55 & 7645 & 4175 & 2575 & 1720 & 1230 & 915 & 720 & 570 \\
\hline 60 & 7120 & 3890 & 2395 & 1605 & 1145 & 845 & 665 & 535 \\
\hline 65 & 5400 & 2945 & 1805 & 1205 & 860 & 650 & 490 & 400 \\
\hline 70 & 1565 & 905 & 605 & 420 & 305 & 235 & 185 & 125 \\
\hline 75 & 65 & 65 & 65 & 65 & 65 & 65 & 65 & 65 \\
\hline 80 & 65 & 65 & 65 & 65 & 65 & 60 & 45 & 35 \\
\hline
\end{tabular}


TABle 7: Network lifetime of EHPS.

\begin{tabular}{|c|c|c|c|c|c|c|c|c|}
\hline \multirow{2}{*}{ Distance $(\mathrm{m})$} & \multicolumn{8}{|c|}{ Rank } \\
\hline & 2 & 3 & 4 & 5 & 6 & 7 & 8 & 9 \\
\hline 10 & 7845 & 4385 & 2640 & 1785 & 1350 & 1015 & 835 & 665 \\
\hline 15 & 7845 & 4385 & 2640 & 1785 & 1350 & 1015 & 835 & 665 \\
\hline 20 & 7845 & 4385 & 2640 & 1785 & 1350 & 1015 & 835 & 665 \\
\hline 25 & 7845 & 4385 & 2640 & 1785 & 1350 & 1015 & 835 & 665 \\
\hline 30 & 7810 & 4265 & 2660 & 1855 & 1385 & 1025 & 820 & 665 \\
\hline 35 & 7810 & 4265 & 2660 & 1855 & 1385 & 1025 & 820 & 665 \\
\hline 40 & 7810 & 4265 & 2660 & 1855 & 1385 & 1025 & 820 & 665 \\
\hline 45 & 7810 & 4265 & 2660 & 1855 & 1385 & 1025 & 820 & 665 \\
\hline 50 & 7805 & 4255 & 2660 & 1855 & 1385 & 1025 & 830 & 675 \\
\hline 55 & 7685 & 4205 & 2620 & 1830 & 1375 & 1025 & 830 & 670 \\
\hline 60 & 7165 & 3960 & 2470 & 1685 & 1335 & 1000 & 780 & 665 \\
\hline 65 & 5455 & 3355 & 2105 & 1440 & 1190 & 905 & 750 & 665 \\
\hline 70 & 2705 & 1385 & 785 & 545 & 545 & 365 & 305 & 305 \\
\hline 75 & 65 & 65 & 65 & 65 & 65 & 65 & 65 & 65 \\
\hline 80 & 65 & 65 & 65 & 65 & 65 & 65 & 65 & 65 \\
\hline
\end{tabular}

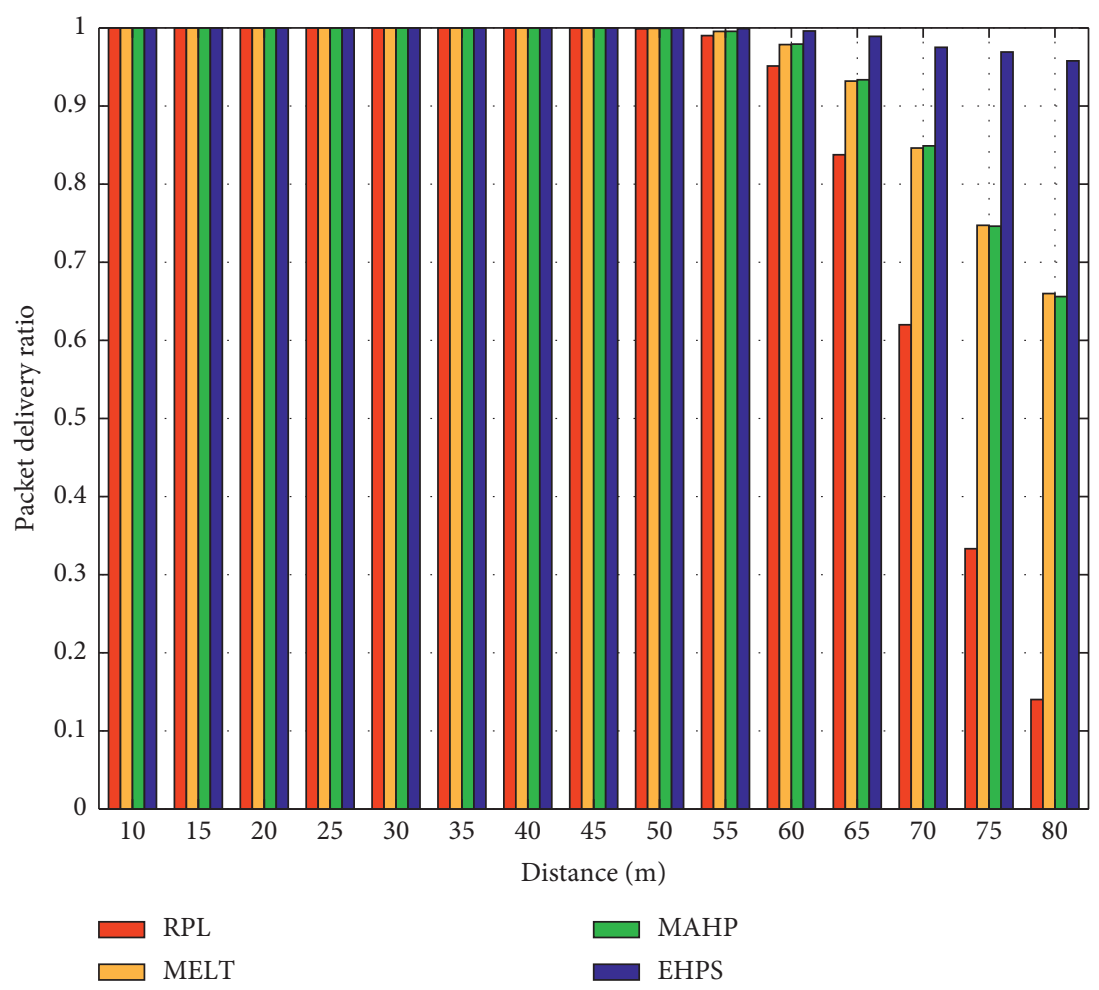

FIgURE 10: Comparison of PDR as a function of distance of household energy usage.

technology. When the distance is lower than $55 \mathrm{~m}$, the multiparent scheme is frequently activated since it consumes less energy than the single parent with cognitive radio. When the distance increases, the expected overall performance of multiparent solution determined by FAHP is lower than that using the cognitive radio. Therefore, the single parent with cognitive radio is more activated. Then, the high PDR can be maintained as much higher than others. As shown in Figure 13, EHPS presents the lowest latency as compared to others for all transmission distance while other techniques can perform data transmission when the distance is lower than $75 \mathrm{~m}$. At $80 \mathrm{~m}$, RPL, MELT, and MAHP cannot forward the data to the parent meter since the PDR is 0 , so their latency values are noticeably high.

From the simulation results, the empowered hybrid parent selection (EHPS) algorithm is flexible to be used under various sizes of data packages and distances. By hybridizing usage of multiparent solution and the single parent with cognitive radio, EHPS maximizes the network lifetime under long data transmission range and presents the highest PDR and lowest latency among others. In our proposed algorithm, we follow the information exchange 


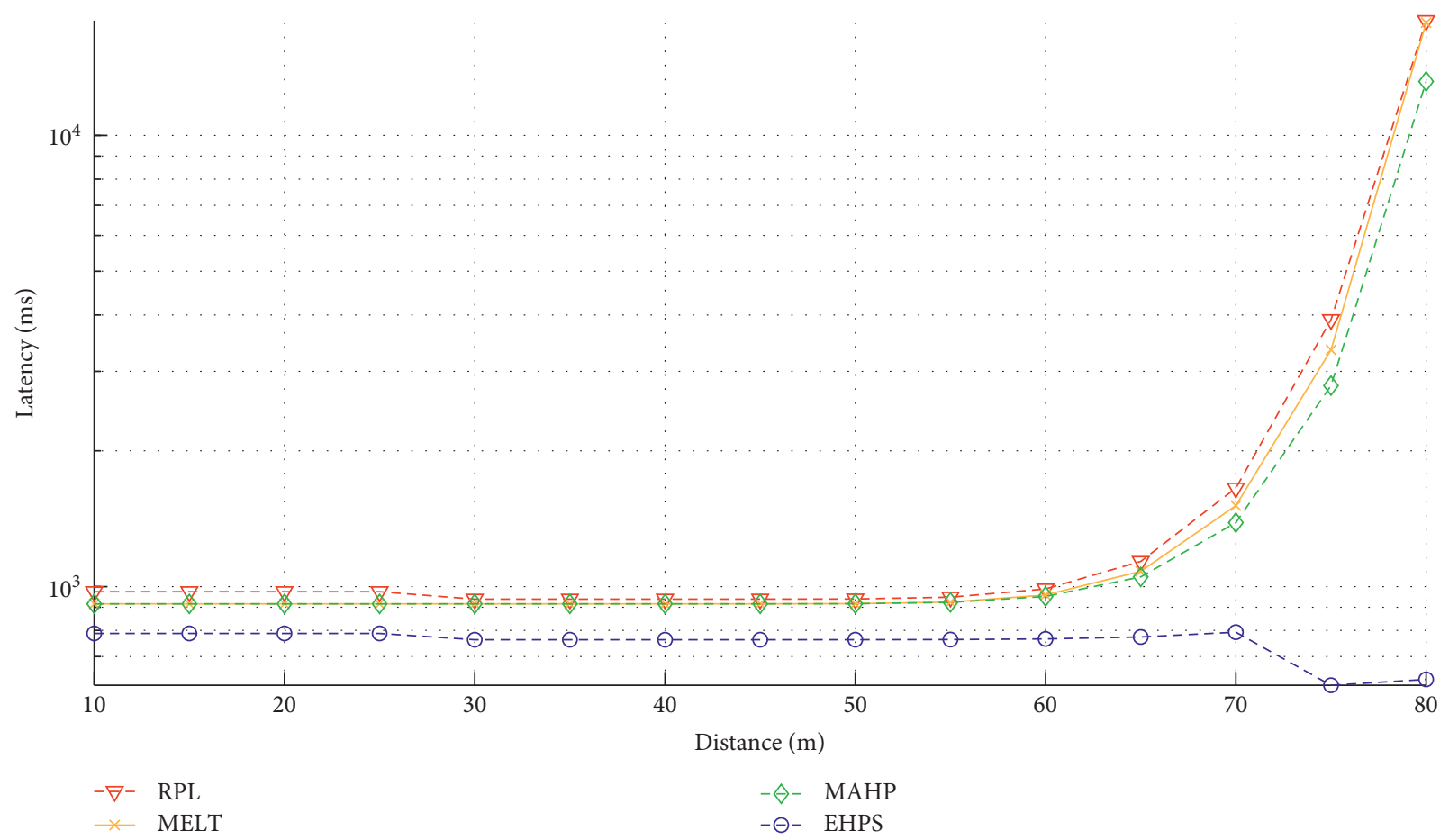

Figure 11: Comparison of latency as a function of distance of household energy usage.

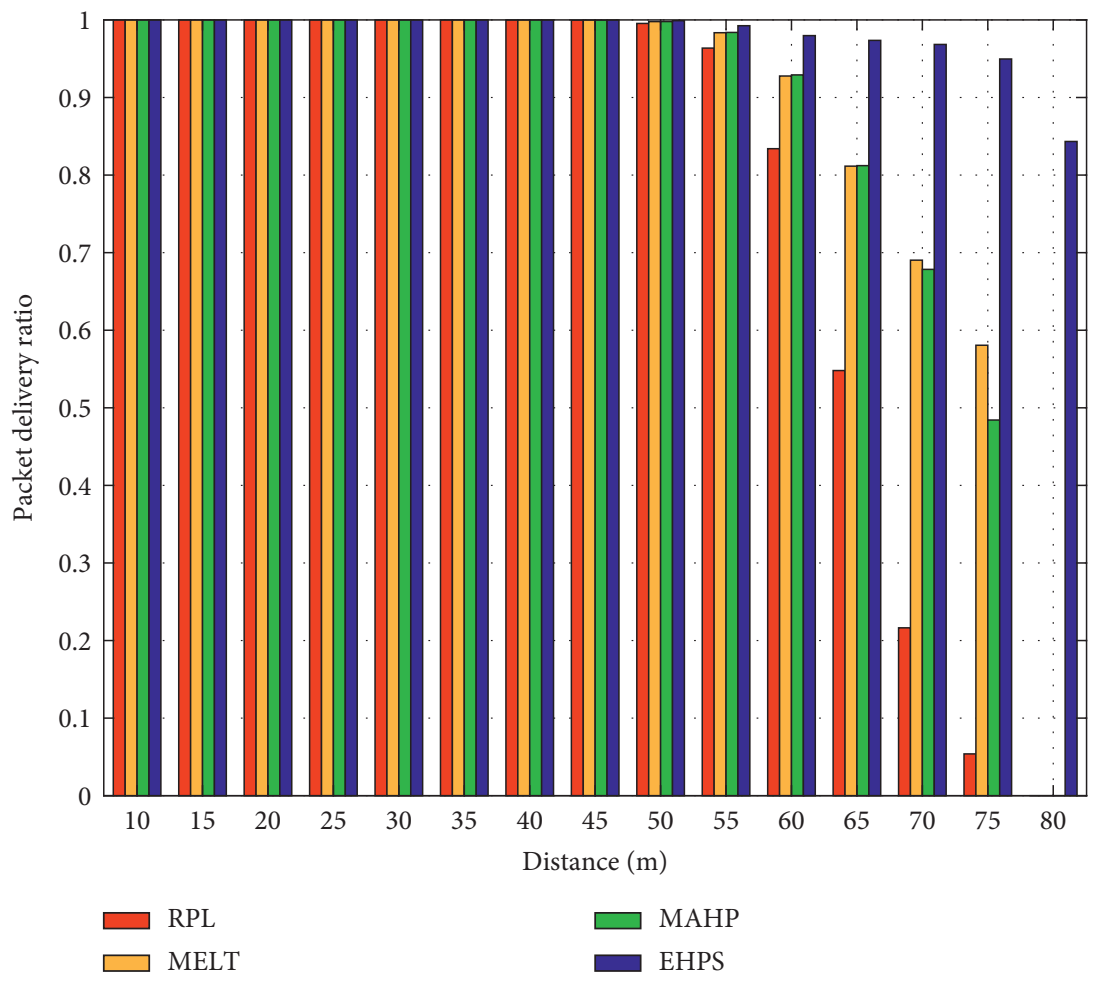

FIGURE 12: Comparison of PDR as a function of distance of manufacturing energy usage.

according to the RPL mechanism where the message-DIO, $\mathrm{DAO}$, and DIS-for route construction and maintenance is exchanged between nodes periodically. Therefore, the extra overhead is not required. On the other hand, the complexity of EHPS is bound at $O\left(n^{3}\right)$ where $n$ is number of candidate parents. Moreover, the EHPS does not suffer from a high complexity of FAHP since the numbers of main criteria and alternatives are low. Moreover, by determining the parents' quality comprehensively through an adaptive FAHP scheme, the workload can be balanced; then, it is facilitated for the 


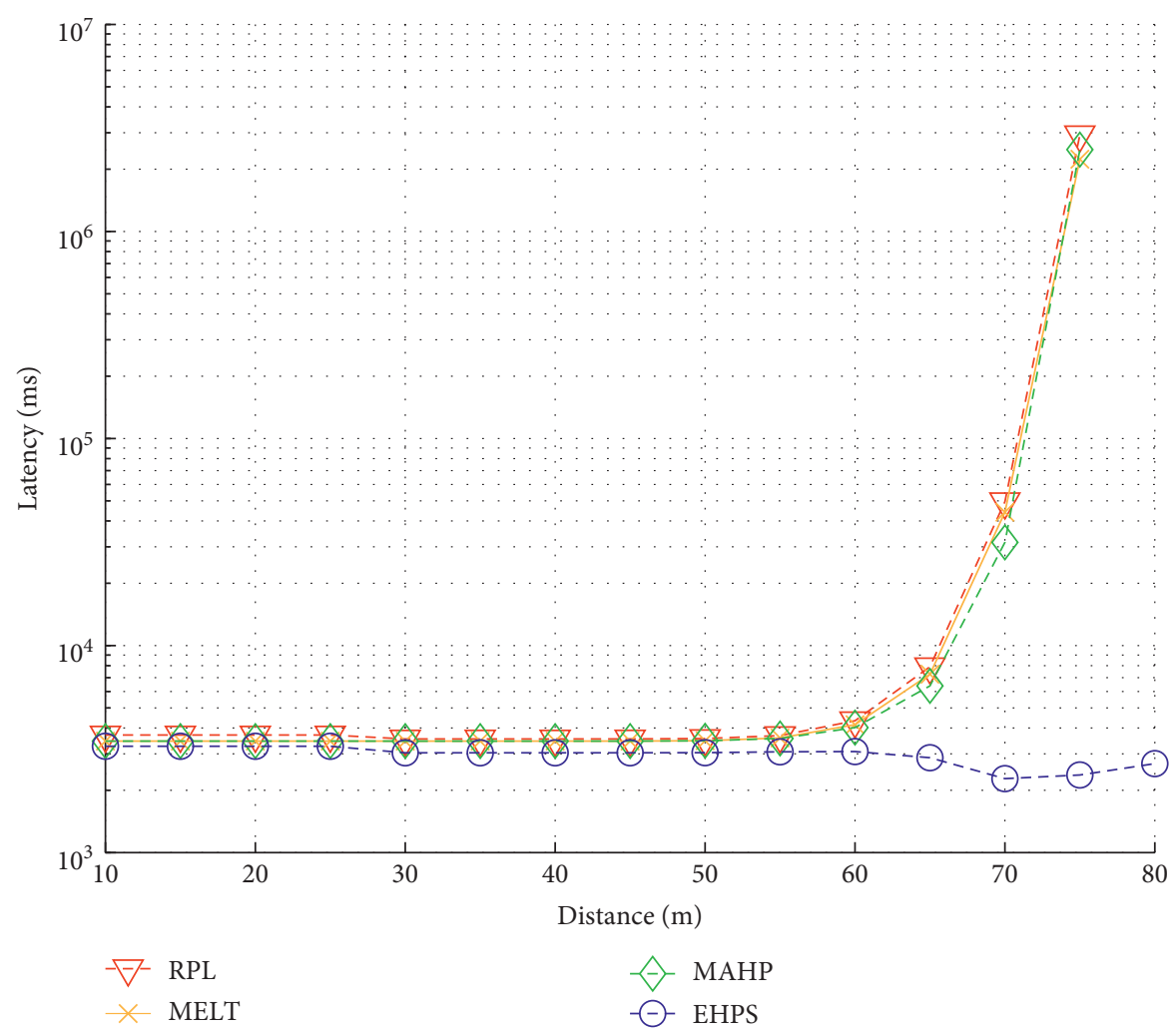

Figure 13: Comparison of latency as a function of distance of manufacturing energy usage.

electrical production planning and electricity billing application.

\section{Conclusion}

In this paper, an "empowered hybrid parent selection (EHPS)" is proposed to overcome the issues of the parent selections of RPL for AMI network. In general, single parent and multiparent solutions suffer from the tradeoff between the network lifetime and transmission performance. Even if the single parent selection presents higher network lifetime than multiparent solutions, it gives worse transmission performance under long transmission range and large data size. On the other hand, multiparent solutions meet high transmission performance but give low network lifetime due to the energy consumption based on multipath strategy. By hybridizing the merits of multiple parents and single parent with cognitive radio technology, EHPS maximizes the network lifetime over long transmission range and large data size. Through the proposed hybrid scheme with adaptive fuzzy analytic hierarchy process (FAHP), EHPS balances the meter workload effectively and is flexible with respect to the transmission range and data size.

\section{Data Availability}

No data were used to support this study.

\section{Conflicts of Interest}

The authors declare that they have no conflicts of interest.

\section{References}

[1] V. C. Gungor, D. Sahin, T. Kocak et al., "Smart grid technologies: communication technologies and standards," IEEE Transactions on Industrial Informatics, vol. 7, no. 4, pp. 529-539, 2011.

[2] V. C. Gungor, D. Sahin, T. Kocak et al., "A survey on smart grid potential applications and communication requirements," IEEE Transactions on Industrial Informatics, vol. 9, no. 1, pp. 28-42, 2013.

[3] U. Ozgur, S. Tonyali, K. Akkaya, and F. Senel, "Comparative evaluation of smart grid AMI networks: performance under privacy," in Proceedings of the 2016 IEEE Symposium on Computers and Communication (ISCC), pp. 1-3, Messina, Italy, June 2016.

[4] S. Nimbargi, S. Mhaisne, S. Nangare, and M. Sinha, "Review on AMI technology for smart meter," in Proceedings of the IEEE International Conference on Advances in Electronics, Communication and Computer Technology (ICAECCT), pp. 21-27, Pune, India, December 2016.

[5] Z. Fan, P. Kulkarni, S. Gormus et al., "Smart grid communications: overview of research challenges, solutions, and standardization activities," IEEE Communications Surveys \& Tutorials, vol. 15, no. 1, pp. 21-38, 2013.

[6] T. Winter, RPL: IPv6 Routing Protocol for Low-Power and Lossy Networks, IETF, Fremont, CA, USA, 2012.

[7] E. Ancilolotti, R. Bruno, and M. Conti, RPL Routing Protocol in Advanced Metering Infrastructure: An Analysis of the Unreliability Problems, IFIP, Laxenburg, Austria, 2012.

[8] I. F. Akyildiz, W. Su, Y. Sankarasubramaniam, and E. Cayirci, "Wireless sensor networks: a survey," Computer Networks, vol. 38, no. 4, pp. 393-422, 2002. 
[9] D. Wang, Z. Tao, J. Zhang, and A. Abouzeid, "RPL based routing for advanced metering infrastructure in smart grid," in Proceedings of the 2010 IEEE International Conference onCommunications Workshops (ICC), pp. 1-6, Cape Town, South Africa, May 2010.

[10] X. Yang, "The application of RPL routing protocol in low power wireless sensor and lossy networks," Sensors \& Transducers, vol. 170, no. 5, pp. 107-111, 2014.

[11] A. Kheaksong, K. Srisomboon, and W. Lee, "A comparative study of various routing protocols for smart grid communication,” ECTI E-Magazine, vol. 10, no. 1, 2016.

[12] A. R. Parnian, M. R. Kharazmi, and R. Javidan, "RPL routing protocol in smart grid communication," ARPN Journal of Systems and Software, vol. 4, no. 5, pp. 128-132, 2014.

[13] J. Tripathi, Performance Evaluation of the Routing Protocol for Low-Power and Lossy Networks (RPL), Cisco Systems, San Jose, CA, USA, 2012.

[14] M. Kuzlu, M. Pipattanasomporn, and S. Rahman, "Communication network requirements for major smart grid applications in HAN, NAN and WAN," Computer Networks, vol. 67, pp. 74-88, 2014.

[15] W. Zhang, G. Han, Y. Feng, and J. Lloret, "IRPL: an energy efficient routing protocol for wireless sensor networks," Journal of Systems Architecture, vol. 75, pp. 35-49, 2017.

[16] P. Thubert, Objective Function Zero for RPL, Cisco Systems, San Jose, CA, USA, 2012.

[17] S. A. Abdel Hakeem, A. A. Hady, and H.W. Kim, "RPL routing protocol performance in smart grid applications based wireless sensors: experimental and simulated analysis," Electronics, vol. 8, no. 2, 2019.

[18] O. Gnawali and P. Levis, RFC 6719-the Minimum Rank with Hysteresis Objective Function, Internet Engineering Task Force (IETF), Fremont, CA, USA, 2012.

[19] A.-L. Kampen, K. Øvsthus, and Ø. Kure, "Energy balancing algorithms in wireless sensor networks," in Proceedings of the 2015 Federated Conference on Computer Science and Information Systems (FedCSIS), vol. 5, pp. 1223-1231, Lodz, Poland, September 2015.

[20] P. Kamgueu, E. Nataf, T. D. Ndie, and O. Faster, Energy-Based Routing Metric for RPL, INRIA, Domaine de Voluceau, France, 2013.

[21] L.-H. Chang, T.-H. Lee, S.-J. Chen, and C.-Y. Liao, "Energyefficient oriented routing algorithm in wireless sensor networks," in Proceedings of the 2013 IEEE International Conference on Systems, Man, and Cybernetics, pp. 3813-3818, Manchester, UK, October 2013.

[22] P. M. Esposito, M. E. M. Campista, I. M. Moraes, L. H. M. K. Costa, O. C. M. B. Duarte, and M. G. Rubinstein, "Implementing the expected transmission time metric for OLSR wireless mesh networks," in Proceedings of the 2008 1st IFIP Wireless Days, Dubai, United Arab Emirates, November 2008.

[23] O. Iova, F. Theoleyre, and T. Noel, "Improving the network lifetime with energy-balancing routing: application to RPL," in Proceedings of the 2014 7th IFIP Wireless and Mobile Networking Conference (WMNC), pp. 1-8, Vilamoura, Portugal, May 2014.

[24] P. Thulasiraman, "RPL routing for multigateway AMI networks under interference constraints," in Proceedings of the 2013 IEEE International Conference on Communications (ICC), Budapest, Hungary, June 2013.

[25] M. Ali Lodhi, A. Rehman, M. M. Khan, and F. B. Hussain, "Multiple path RPL for low power lossy networks," in
Proceedings of the 2015 IEEE Asia Pacific Conference on Wireless and Mobile (APWiMob), Bandung, Indonesia, August 2015 .

[26] O. Iova, F. Theoleyre, and T. Noel, "Using multiparent routing in RPL to increase the stability and the lifetime of the network," Ad Hoc Networks, vol. 29, 2015.

[27] A. H. Process, "The analytic hierarchy process," Computing, vol. 18 , no. 2, 1980.

[28] K. Teknomo, "Analytic hierarchy process (AHP) tutorial," 2006, http://people.revoledu.com/kardi/tutorial/AHP/AHP. htm.

[29] Y. Min, L. T. Yang, F. Wang, and W. Wang, "Dynamic sleeping algorithm based on AHP for wireless sensor networks," in Proceedings of the 2008 Second International Conference on Future Generation Communication and Networking, pp. 387-392, Hainan, China, December 2008.

[30] G. Di Bona and A. Forcina, "Analytic critical flow method (ACFM): a reliability allocation method based on analytic hierarchy process," Journal of Failure Analysis and Prevention Volume, vol. 17, no. 1, pp. 1-15, 2017.

[31] G. Di Bona, D. Falcone, A. Forcina, A. Silvestri, and L. Silvestri, "A new model for maintenance strategy based on failure analysis and multicriteria approach," International Journal of Information Systems in the Service Sector (IJISSS), vol. 12, no. 4, pp. 60-90, 2020.

[32] W. Alayed, L. Mackenzie, and D. Pezaros, "Analytical hierarchy process multi-metric objective function for RPL," in Proceedings of the IEEE 17th International Symposium on Network Computing and Applications (NCA), Cambridge, MA, USA, November 2018.

[33] G. Kabir and M. Ahsan Akhtar Hasin, "Comparative analysis of AHP and Fuzzy AHP models for multicriteria inventory classification," International Journal of Fuzzy Logic Systems (IJFLS), vol. 1, 2011.

[34] S. Paul, A. Chakraborty, and J. S. Banerjee, "A fuzzy AHPbased relay node selection protocol for wireless body area networks (WBAN)," in Proceedings of the 2017 4th International Conference on Opto-Electronics and Applied Optics (Optronix), Kolkata, India, November 2017.

[35] M. Imran Tariq, "Prioritization of information security controls through fuzzy AHP for cloud computing networks and wireless sensor networks," Sensors, vol. 20, no. 5, pp. 1-39, 2020.

[36] Y.-C. Liang, K.-C. Chen, G. Y. Li, and P. Mähönen, “Cognitive radio networking and communications: an overview," IEEE Transactions on Vehicular Technology, vol. 60, no. 7, 2011.

[37] Z. Qin, "20 years of evolution from cognitive to intelligent communications," IEEE Transactions on Cognitive Communication and Networking, vol. 6, no. 1, 2020.

[38] CC2500, "Errata Notes (Rev. E)," 2013, https://www.ti.com/ lit/er/swrz002e/swrz002e.pdf. 